\title{
SENSITIVITY OF HIGH-RESOLUTION SIMULATIONS OF HURRICANE BOB (1991) TO PLANETARY BOUNDARY LAYER PARAMETERIZATIONS
}

\author{
Scott A. Braun and Wei-Kuo Tao \\ Laboratory for Atmospheres, NASA/Goddard Space Flight Center, Greenbelt, MD
}

submitted to

Monthly Weather Review

October 4, 1999

Corresponding Author: Dr. Scott A. Braun, Mesoscale Atmospheric Processes Branch, NASA/GSFC, Code 912 , Greenbelt, MD 20771.braun@gilbert.gsfc.nasa.gov. 


\section{ABSTRACT}

The MM5 mesoscale model is used to simulate Hurricane Bob (1991) using grids nested to high resolution $(4 \mathrm{~km})$. Tests are conducted to determine the sensitivity of the simulation to the available planetary boundary layer parameterizations, including the bulk-aerodynamic, Blackadar, Medium-Range Forecast (MRF) model, and Burk-Thompson boundary-layer schemes. Significant sensitivity is seen, with minimum central pressures varying by up to $17 \mathrm{mb}$. The Burk-Thompson and bulk-aerodynamic boundary-layer schemes produced the strongest storms while the MRF scheme produced the weakest storm. Precipitation structure of the simulated hurricanes also varied substantially with the boundary layer parameterizations. Diagnostics of boundary-layer variables indicated that the intensity of the simulated hurricanes generally increased as the ratio of the surface exchange coefficients for heat and momentum, $C_{h} / C_{M}$, although the manner in which the vertical mixing takes place was also important. Findings specific to the boundary-layer schemes include: 1) the MRF scheme produces mixing that is too deep and causes drying of the lower boundary layer in the inner-core region of the hurricane; 2) the bulk-aerodynamic scheme produces mixing that is probably too shallow, but results in a strong hurricane because of a large value of $\left.C_{h} / C_{M}(\sim 1.3) ; 3\right)$ the MRF and Blackadar schemes are weak partly because of smaller surface moisture fluxes that result in a reduced value of $\left.C_{h} / C_{M}(\sim 0.7) ; 4\right)$ the Burk-Thompson scheme produces a strong storm with $C_{h} / C_{M} \sim 1$; and 5) the formulation of the wind-speed dependence of the surface roughness parameter, $z_{0}$, is important for getting appropriate values of the surface exchange coefficients in hurricanes based upon current estimates of these parameters. 


\section{Introduction}

Observations within the atmospheric boundary layer in the inner-core region of hurricanes are rare, often available only from isolated dropsondes or buoys. This lack of data forces modelers to use boundary layer parameterizations that have largely been developed for lower wind speed conditions. Assumptions about boundary layer processes are particularly important to models attempting to simulate the convective-scale to mesoscale processes responsible for the evolution and maintenance of hurricanes. It is important to understand how assumptions regarding the character of surface fluxes and vertical mixing within the boundary layer impact simulations of hurricanes so that we understand the limitations of current assumptions and have some direction for future observational studies. This study describes highresolution simulations of Hurricane Bob (1991) and their sensitivity to different formulations of boundary layer processes, and provides detailed analysis of the components of these boundary layer schemes that produce the sensitivity.

Surface fluxes of sensible and latent heat play a vital role in the development and maintenance of tropical cyclones (Byers, 1944). Riehl (1954), Palmén and Riehl (1957), and Malkus and Riehl (1960) have noted that while the heat gained from the ocean is only a small fraction of that transported inward by the radial inflow or released by condensation in the updrafts, it is essential for growth of the hurricane. Malkus and Riehl (1960) showed that surface fluxes in the inner core of hurricanes are capable of increasing the equivalent potential temperature, $\theta_{\varepsilon}$, by more than $10 \mathrm{~K}$, which contributes significantly to the deepening of hurricanes to pressures well below $1000 \mathrm{mb}$. Ooyama (1969) suggested that surface fluxes in the outer region of hurricanes are also necessary in order to maintain $\theta_{e}$ against the effects of entrainment of subsiding dry air into the planetary boundary layer (PBL). Emanuel (1986) and 
Rotunno and Emanuel (1987) further demonstrated the importance of surface fluxes by showing that hurricanes can develop and be maintained even in environments with no initial convective available potential energy as a result of energy derived from surface fluxes of sensible and latent heat.

The dependence of the maximum tangential winds on the surface drag coefficients for heat and momentum was first suggested by Malkus and Riehl (1960). Ooyama (1969), Rosenthal (1971), and Emanuel (1986, 1995, 1997), using numerical and mathematical models of hurricanes, confirmed that the potential intensity of hurricanes increases (decreases) with increases in the drag coefficient for heat (momentum). In other words, hurricanes become stronger as the transfer of sensible and latent heat from the sea surface is increased and as the frictional dissipation is decreased. These models treated the PBL as a single layer and did not consider the impact of how the vertical mixing takes place.

Anthes and Chang (1978) conducted simulations of hurricanes using a model with high vertical resolution in the boundary layer, but relatively coarse resolution above the PBL and a coarse $60-\mathrm{km}$ horizontal resolution. By comparing the model with high resolution in the PBL (a 9-level model) to one treating the PBL as a single layer (a 5-level model), they found that the extra degrees of freedom allowed by resolving the boundary layer impacted the behavior of the simulated storms and their sensitivity to changes in surface properties, although their structures above the PBL were similar. Specifically, differences in the responses of the 9- and 5-level models to changes in sea-surface temperature (SST) included: no initial adjustment of the winds, a stronger response to SST, a nonlinear variation of intensity with SST, weaker dynamic coupling, and a weaker change in evaporation in the 5-level model. 
The different behaviors of the 9- and 5-level models raise questions about the sensitivity of more sophisticated, higher-resolution models to the parameterization of PBL processes. Furthermore, the dependence of potential hurricane intensity on surface exchange coefficients for heat and momentum suggests that, for PBL schemes that utilize different parameterizations of surface fluxes, some PBL schemes may be more predisposed toward developing strong hurricanes than others. As model resolution approaches the convective scale, how sensitive will simulations be to different formulations of the PBL physics? Will the sensitivity to the PBL physics be primarily a function of the surface flux parameterization, or will the parameterization of the vertical fluxes above the surface play an important role?

The Penn State University—National Center for Atmospheric Research (PSU-NCAR) MM5 mesoscale model has already been shown to have some skill at simulating hurricanes (Karyampudi et al. 1998) including some at high $(<6 \mathrm{~km})$ resolution (Liu et al. 1997). The model contains several different representations of PBL processes including a simple bulk-aerodynamic PBL (Deardorff 1972), the Blackadar PBL (Blackadar 1976, 1979; Zhang and Anthes 1982; Oncley and Dudhia 1995), a version of the Medium-Range Forecast (MRF) model PBL (Hong and Pan 1996), and a version of the turbulent kinetic energy (TKE) predicting scheme of Burk and Thompson (1989). Evaluation of the model PBL physics can provide insight into the sensitivity of hurricane simulations to the representations of vertical mixing and surface fluxes. In this study, we perform such sensitivity tests in simulations of Hurricane Bob (1991). In section 2, the numerical experiments are described, including the derivation of initial conditions and modifications to some of the model physics. Section 3 provides a verification of one of the simulations using available observations, while section 4 describes the results of the sensitivity tests. Brief descriptions of the PBL and surface physics are given in Appendices A and B. 


\section{Simulation description}

The model used in this study is the PSU—NCAR nonhydrostatic mesoscale model MM5 (V2.5; Dudhia 1993; Grell et al. 1995). MM5 was used to conduct 72-h simulations of Hurricane Bob (0000 UTC 16 August - 0000 UTC 19 August, 1991) using a coarse grid consisting of $193 \times 163$ grid points in $x, y$ with a grid spacing of $36-\mathrm{km}$ (see Fig. 1). Higher resolution simulations were performed using a one-way interacting nest between the coarse grid and two finer grids (12 and $4 \mathrm{~km}$, indicated in Fig. 1). The grid meshes included 27 vertical half- $\sigma$ levels', where $\sigma$ is defined as $\sigma=\left(p-p_{\text {top }}\right) /\left(p_{\text {sfc }}-p_{\text {top }}\right), p$ is pressure, and $p_{\text {sfc }}$ and $p_{\text {top }}(25 \mathrm{mb})$ are the pressures at the surface and model top, respectively.

The coarse grid was centered at $33^{\circ} \mathrm{N}, 84^{\circ} \mathrm{W}$. Initial and boundary conditions were obtained from 12-hourly global analyses from the European Center for Medium-Range Weather Forecasts (ECMWF) archived at NCAR. Analysis fields, including temperature, relative humidity, geopotential height, and winds at mandatory pressure levels and with horizontal resolution of $2.5^{\circ} \times 2.5^{\circ}$, were interpolated horizontally to model grid points. These interpolated analyses were refined by adding information from standard twice daily rawinsondes and 3-hourly surface and buoy reports using a Barnes objective analysis technique (Manning and Haagenson, 1992). Final analyses were then interpolated to the model $\sigma$ levels.

No special observations were available near the initial time, and inserting a bogus vortex was not practical since the storm was only in the tropical depression stage at the initial time. To avoid the impression that a good simulation for the coarse mesh was obtained easily, it should be noted that use of analyses from the National Center for Environmental Prediction (NCEP) for

\footnotetext{
${ }^{1}$ The model full- $\sigma$ levels are at $1 ., 0.99,0.98,0.96,0.93$, and then decrease to 0.05 at 0.04 intervals, followed by the last level at $\sigma=0$. Half- $\sigma$ levels are located midway between the full- $\sigma$ levels.
} 
initial and boundary conditions failed to produce a hurricane. Furthermore, a hurricane was only obtained when using the Betts-Miller cumulus parameterization. The Kain-Fritsch and Grell schemes did not produce hurricanes.

Physics options for the coarse-grid run included the Betts-Miller cumulus parameterization, the Goddard Cumulus Ensemble (GCE) model cloud microphysics (Tao and Simpson 1993), the MRF PBL scheme (Hong and Pan 1996), and the cloud radiative scheme of Dudhia (1989). The Goddard microphysical scheme was modified to allow for the option to use graupel or hail as a third class of ice. The modifications included changes to the particle distribution, density, and fall speed constants as well as to the transformation rates, which had previously been written explicitly in terms of the hail fall speed parameters.

High-resolution simulations were conducted by using 1 -h output from the $36-\mathrm{km}$ grid to provide initial and boundary conditions for the 12 and $4-\mathrm{km}$ grids ( $163 \times 178$ grid points) beginning at hour 48 of the 72 -h control run (thus providing identical initial conditions for all experiments). The 4-km domain was moved with the storm in order to keep the storm nearly centered within the domain. Physics options for the 4-km control simulations were similar to the coarse-grid simulation except that no cumulus parameterization was used on the 4-km grid.

For the high-resolution simulations, modifications were made to the microphysics that included the addition of subroutines that maintain total water balance while eliminating negative mixing ratios that arise after the calculation of advection terms, changing the slope and intercept parameters in the Fletcher equation (which specifies the number concentration of ice nuclei as a function of temperature) from 0.6 and $10^{-5} \mathrm{~L}^{-1}$ to 0.46 and $10^{-3} \mathrm{~L}^{-1}$, respectively, in order to provide a better fit to observed ice concentrations (Meyers et al. 1992), limiting the ice nuclei concentration to $\sim 1000 \mathrm{~L}^{-1}$, and adding a small fall velocity for cloud ice of $0.2 \mathrm{~m} \mathrm{~s}^{-1}$ (Braun et al. 
1999). A new formulation of the conversion of cloud ice to snow by vapor deposition developed by Braun et al. (1999) was incorporated to correct for a lack of a dependence of this process on relative humidity. The original GCE scheme used a saturation adjustment technique that involved both cloud water and cloud ice. In the modified GCE scheme, the saturation adjustment involves cloud water only. Intercept parameters for rain, snow and graupel were set to $22 \times 10^{6}$ $\mathrm{m}^{-4}, 100 \times 10^{6} \mathrm{~m}^{-4}, 4 \times 10^{6} \mathrm{~m}^{-4}$, respectively. The rain intercept is same as that used by Lord et al. (1984) in simulations of hurricanes. For snow, the intercept parameter was based on simulations of convective systems by Ferrier et al. (1995). The graupel value is the standard value in the model and is based upon Rutledge and Hobbs (1984).

Four simulations were conducted to test the sensitivity to PBL physics. For these highresolution runs, the MRF PBL scheme, the Blackadar scheme, the bulk-aerodynamic scheme, and the Burk-Thompson scheme were used. A brief review of each of the PBL schemes is given in Appendices A and B.

\section{Verification}

In this section, simulation results from both the coarse and fine grids are compared to available observations. These observations include time series of storm track and minimum central pressure, radar reflectivity fields and flight-level winds from reconnaissance aircraft, NEXRAD radar reflectivities at Cape Hatteras, North Carolina, and available surface wind analyses from the Hurricane Research Division of the National Oceanic and Atmospheric Administration (NOAA). Unfortunately, vertical structure information from the tail Doppler radars of NOAA's WP-3D reconnaissance aircraft were not available as a result of equipment 
failures. Therefore, the vertical structure of the simulated storm cannot be verified, and the quality of the simulation must be judged strictly from the low-level horizontal fields.

Since multiple fine-grid simulations were conducted, we select one case for verification purposes, that one being the case that most closely resembles the observed storm evolution. This case is the simulation that uses the Burk-Thompson PBL scheme. The remaining simulations will be examined in later sections to demonstrate the sensitivity of the results to model physics.

\section{a. Coarse-grid results}

Bob was a tropical depression at 0000 UTC 16 August and became a hurricane by 1800 UTC 17 August. Its central pressure was observed to fall steadily from an initial value of 1012 $\mathrm{mb}$ to its minimum value of $950 \mathrm{mb}$ by 0600 UTC 19 August. The simulated storm was initially poorly defined and unorganized, located northwestward of the observed location, and for the first $24 \mathrm{~h}$ of simulation, remained relatively stationary (see inset in Fig. 1). After $\sim 24$ h of simulation, the vortex became more organized and approximately coincided with the observed storm location, primarily as a result of the observed storm "catching up" to the simulated one. Following this time, the simulated storm moved northward at a speed comparable to the observed storm, with the track (Fig. 1, dashed line) generally lying $1^{\circ}$ eastward of the observed track during the latter $36 \mathrm{~h}$ of the simulation.

Figure 2 shows time series of the observed central pressures and the simulated central pressures for the $72-\mathrm{h}$ period of the coarse-grid simulation. The observed central pressure started at $1014 \mathrm{mb}$, decreased slowly between $0-36 \mathrm{~h}$, and then decreased more rapidly after $36 \mathrm{~h}$, reaching a value of $957 \mathrm{mb}$ by $72 \mathrm{~h}$. Note that the storm continued to deepen to $950 \mathrm{mb}$ by 0600

UTC on 19 August, six hours after the end of the simulation period. The coarse $36-\mathrm{km}$ grid produced a central pressure decrease from $1015 \mathrm{mb}$ to $982 \mathrm{mb}$. The under-prediction of the 
central pressure decrease is due in part to the poor representation of the vortex in the model initial conditions. However, a weaker storm should also be expected to some extent because the $36-\mathrm{km}$ resolution is unlikely to be able to represent the very small scales over which the pressure changes near the storm center.

\section{b. Fine-grid results}

The evolution of the simulated central pressure from Burk-Thompson PBL case is also shown in Fig. 2 starting at 48 h. During the initial 6-8 h, the central pressure decreases rapidly as a result of spin-up of the vortex on the fine grid. This spin-up phase involves an adjustment (contraction) of the vortex caused by the increase in resolution from 36 to $4 \mathrm{~km}$ as well as spinup of the cloud microphysics as precipitation processes switch from being represented by the cumulus parameterization to a bulk microphysical parameterization. Following the spin-up period, the rate of pressure fall corresponds fairly closely with the observed rate.

The precipitation structure of Hurricane Bob as seen from the Cape Hatteras NEXRAD radar is shown in Fig. 3a. The radar indicates a partial eye wall, open to the south, at a radius of about $25 \mathrm{~km}$. This partial eye wall structure was persistent throughout much of the time for which radar observations were available (two lower-fuselage radar images from the NOAA WP3D aircraft, not shown, at 2000 UTC 17 August and 0500 UTC 18 August, plus NEXRAD images from 2345 UTC 18 August to 0445 UTC 19 August). Outer rain bands on the eastern side of the storm spiral inward toward the northern side, where they merge with a region of heavy, mostly stratiform precipitation northwest of the eye. A convective band with reflectivities exceeding $50 \mathrm{dBZ}$ extends from north of the center eastward at a radius of about $160-200 \mathrm{~km}$, and another band of weaker precipitation extends from the southern end around to the northwestern side of the storm at a radius of about $80-100 \mathrm{~km}$. 
The simulated radar reflectivity field at $72 \mathrm{~h}$ (Fig. 3b), valid at 0000 UTC 19 August (about 1 hour prior to the radar image), shows a well defined eye wall. The eye wall contains high reflectivities encircling most of the eye, typical of much of the simulation period. While the model generally fails to produce the partial eye wall, it frequently produces a region of weak reflectivities in the eye wall, often along the southern end of the storm. The simulated eye-wall radius is about $40-45 \mathrm{~km}$, about twice as wide as observed. Precipitation outside of the eye wall consists of weakly organized cells rather than well-organized bands or wide stratiform rain areas. Although the simulated eye wall radius is larger than observed, the overall size of the precipitation area is smaller. Along the eastern side of the simulated storm, the eye-wall rain bands protrude southward in a manner similar to the observed bands in this area. A poorly defined band extends from the southern end of the storm back towards the western side in a manner that resembles the observations, but does not extend far enough northward. While the model produces a broader region of precipitation to the northwest of the center, similar to the observations, the coverage of stratiform precipitation appears to be less than observed. The strong convective band observed about $180 \mathrm{~km}$ to the north of the eye is not simulated at all. An experiment with a $1.3-\mathrm{km}$ grid centered on the storm, to be reported on in a future paper, produced a well-defined convective rain band to the north and northeast of the center, suggesting that higher horizontal resolution was necessary to reproduce the weakly forced convection in some of the outer rain bands.

A surface (10-m) wind analysis valid for 0300 UTC 19 August (provided courtesy of Sam Houston of NOAA/HRD; see Houston and Powell, 1993) is shown in Fig. 4a. The eye was located just east of Cape Hatteras. Maximum winds in excess of $50 \mathrm{~m} \mathrm{~s}^{-1}$ were analyzed to the east of the center. The region of winds exceeding $35 \mathrm{~m} \mathrm{~s}^{-1}$ extended about $2^{\circ}$ of longitude to the 
east of the center, but only $0.5-1^{\circ}$ to the west, suggestive of the impacts of the land surface. A significant contribution to the wide area of winds (and the maximum winds) to the east of the center came from the $10 \mathrm{~m} \mathrm{~s}^{-1}$ northward motion of the hurricane at this time. Simulated winds at the lowest model level $(42 \mathrm{~m})$ are shown in Fig. $4 \mathrm{~b}$. The eye is located about $120 \mathrm{~km}$ southeast of the observed location. Maximum winds slightly exceed $55 \mathrm{~m} \mathrm{~s}^{-1}$ in the southern and eastern portions of the eye wall, in fairly good agreement with the analyzed winds. However, the area of winds exceeding $35 \mathrm{~m} \mathrm{~s}^{-1}$ is only about half as wide as observed. The simulated storm moves northward more slowly than observed $\left(6 \mathrm{~m} \mathrm{~s}^{-1}\right.$ vs. $\left.10 \mathrm{~m} \mathrm{~s}^{-1}\right)$, which contributes to some extent to the smaller area of strong winds to the east of the center.

Flight-level winds from Air Force reconnaissance aircraft (not shown) suggest maximum 850- and 700-mb level winds around 0000 UTC 19 August of about 50-55 $\mathrm{m} \mathrm{s}^{-1}$, implying relatively little vertical variation of the winds at low levels. The model, in contrast, produces maximum winds (not shown) at $850 \mathrm{mb}$ that exceed $70 \mathrm{~m} \mathrm{~s}^{-1}$, while at $700 \mathrm{mb}$ the winds are less than $60 \mathrm{~m} \mathrm{~s}^{-1}$, in closer agreement with the observations. Therefore, the model appears to generate excessive wind speeds just above the boundary layer.

The above verification suggests that the intensity of the storm in terms of minimum sealevel pressure, and to some extent maximum winds, is reasonably well simulated. The simulated eye wall is too large compared to observations, but it is probably unrealistic to expect a 24-h simulation to reproduce eye-wall structures that have evolved over several days. The underprediction of the area of high winds is likely related to the similar under-prediction of the precipitation area, and may be caused by many factors including model initial conditions, inadequate horizontal resolution (for resolving the initiation and maintenance of convective updrafts in the outer regions), and inadequate cloud microphysics and PBL physics. 


\section{Sensitivity to PBL parameterization}

\section{a. Comparison of $P B L$ cases}

Hurricanes rely on air-sea exchanges and PBL processes to provide much of the energy needed for development. Hence, it is reasonable to expect sensitivity to the parameterization of PBL processes. The magnitude of this sensitivity is demonstrated in Fig. 5, which shows time series of central pressure for each of the different PBL cases. Considerable variation in final central pressures is evident, with $17 \mathrm{mb}$ separating the extreme cases. The Burk-Thompson and bulk-aerodynamic PBL schemes produce steady rates of pressure fall throughout most of the simulations, with the Burk-Thompson PBL case generally producing slightly lower pressures. Following the initial spin-up of the storm, the central pressures in the Burk-Thompson and bulkaerodynamic cases are usually within about $3 \mathrm{mb}$ of the observed 6-hourly pressures. The Blackadar and MRF PBL cases tend to result in much weaker storms, with final central pressures that are about 12 and $17 \mathrm{mb}$ weaker, respectively, than in the Burk-Thompson case.

Along with storm intensity, the PBL parameterizations also impact the precipitation structure. In Figure 6, the horizontal structure of the simulated radar reflectivities at $72 \mathrm{~h}$ for the different PBL runs are compared. The Burk-Thompson case (Fig. 6a) was described in the previous section. It generally results in the most compact rain area of the four experiments. For the bulk-aerodynamic PBL (Fig. 6b), heavy precipitation encloses only about half to threefourths of the eye. This precipitation structure more closely resembles observed reflectivities (Fig. 3a) from the Cape Hatteras radar at this time, but only develops within the final hours of the simulation. Convection in outer rain bands to the north and northeast of the center is located at radii of $\sim 150-200 \mathrm{~km}$, in reasonable agreement with the radar observations. When the Blackadar PBL scheme is used (Fig. 6c), precipitation encloses the eye. Outside of the eye wall, a rainband 
extends outward into the southeast quadrant, but otherwise convection is isolated and sparse. The precipitation area is somewhat larger than that in the Burk-Thompson case. For the MRF PBL case (Fig. 6d), heavy rain surrounds the eye and significant outer-band precipitation is present. This case yields the largest areal coverage of precipitation, but also the weakest storm. While it may be argued that cooling and drying of the PBL by convective downdrafts in the outer regions may have contributed to the weaker intensity in this case, it will be shown later in this section that the PBL physics alone can account for the weaker intensity. In general, the variability in the horizontal precipitation structure seen between the different PBL cases is equal to or greater than that obtained from a set of simulations using variable cloud microphysics (not shown), which suggests that quantitative precipitation forecasting in hurricanes can be just as dependent on accurate representation of $\mathrm{PBL}$ processes as it is on accurate representation of cloud microphysics.

The vertical structures of the simulated hurricanes are compared by examining vertical cross sections of the temporally and azimuthally averaged fields for each case for the period 60$66 \mathrm{~h}$ using output every $15 \mathrm{~min}$. Cross sections of vertical velocity are shown in Fig. 7 and indicate that vertical motions in the eye wall are strongest in the Burk-Thompson case (Fig. 7a) and weakest in the bulk-aerodynamic and MRF cases (Figs. $7 b, d$ ). In each case, there is an outward slope of the mean updraft with height. In the Blackadar and Burk-Thompson PBL cases, the updrafts tilt outward very sharply in the lowest few kilometers, but have much smaller tilts at mid-to-upper levels. A similar structure of the vertical motions can be seen in the analyses of Black et al. (1994) in Hurricane Emily (1987) in the right and left front quadrants of the storm (the rear quadrants were characterized by weak or descending motion). In all but the MRF PBL case, relatively strong downdrafts are adjacent to the eye wall at low levels. 
The intensity of the average tangential winds (Fig. 8) follows that of the central pressure depression, i.e., the lower the central pressure, the stronger the mean tangential winds. Consequently, the bulk-aerodynamic and Burk-Thompson PBL cases have the strongest winds while the MRF PBL case contains the weakest winds. Although the vertical motions in the bulkaerodynamic case are relatively weak like those of the MRF case, the tangential winds are as strong as in the Burk-Thompson case. This result suggests that, in the model, the intensity of the hurricane in terms of its tangential winds is not directly related to the intensity of the vertical motions in the eye wall, but also depends upon other factors such as momentum dissipation near the surface (PBL physics).

Radial velocities in the MRF case (Fig. 9d) show relatively deeper, but weaker radial inflow compared to the other cases (Fig. 9a-c). In contrast, the other cases produce very shallow $(<1.5 \mathrm{~km})$ and strong inflow. Nearly coincident with the eye-wall updraft and immediately above the low-level inflow is a region of outflow, which is strongest in the Burk-Thompson case, and weaker in the Blackadar and bulk-aerodynamic cases. This outflow region is similar to that seen in the analysis of Hurricane Allen (1980) by Jorgensen (1984) and in the simulation of Hurricane Andrew by Lui et al. (1997). The divergence that occurs inward of this outflow region contributes to the downward motion along the inside edge of the updraft. At upper levels (8-14 $\mathrm{km}$ altitude), there is considerable variation in the outflow structures. The outflow in the BurkThompson case is quite strong and is maximum within about $100 \mathrm{~km}$ from the center, likely as a result of the much stronger and more upright updraft in that case. The outflow is weaker in the other cases and is generally maximum at larger radii. Evident in the Burk-Thompson case, and to a lesser degree in the Blackadar and bulk-aerodynamic PBL cases, is a secondary outflow layer near $10.5 \mathrm{~km}$ altitude. 
In working towards an understanding of the mechanisms that account for the variations in intensity between the different PBL cases, we start with an examination of the equivalent potential temperature $\left(\theta_{e}\right)$ structure in each case. The maximum potential intensity of hurricanes has been related to the magnitude of the boundary-layer $\theta_{e}$ (Riehl 1954; Malkus and Riehl 1960; Emanuel 1986; Holland 1997), which typically increases radially inward as inflowing air moves toward lower pressure and picks up heat and moisture from the sea surface. The average lowlevel $\theta_{e}$ and cloud water structure for each case is depicted in Fig. 10. Outside of the eye wall ( $r$ $>50 \mathrm{~km}), \theta_{\varepsilon}$ in the boundary layer is comparable in all cases except the MRF PBL case, which is significantly drier. Because of the dry PBL in the MRF case, the cloud base is high, with an average height of about $1 \mathrm{~km}$ outside of the eye wall and about $500-700 \mathrm{~m}$ in the eye wall. In contrast to the MRF case, each of the other cases (Figs. 10a-c) is characterized by relatively low cloud bases, typically about $400-500 \mathrm{~m}$ outside of the eye wall and 100-200 $\mathrm{m}$ in the eye wall. These lower cloud bases are in better agreement with observed and diagnosed cloud bases (Riehl 1954; Malkus and Riehl 1960; Hawkins and Imbembo 1976; Moss and Rosenthal 1975). In the eye-wall region, $\theta_{e}$ increases rapidly towards the center in each case. The Burk-Thompson case (Fig. 10a) is characterized by the highest $\theta_{e}$ within the eye-wall updrafts, with average values up to $352-354 \mathrm{~K}$ between $2-6 \mathrm{~km}$. The other three cases show maximum $\theta_{e}$ in the eye wall of about $350 \mathrm{~K}$, giving the appearance that the differences in intensity cannot be explained by differences in $\theta_{e}$. However, the azimuthally averaged $\theta_{e}$ fields can be misleading if there are asymmetries in the $\theta_{e}$ field or if the high- $\theta_{e}$ air is confined to small convective cores that cover only a fraction of the eye-wall area.

In order to distinguish the thermodynamic characteristics of the air rising within the eye wall in each case, contoured frequency diagrams of $\theta_{e}$ as a function of vertical velocity are 
shown in Fig. 11. These diagrams, similar to the contoured frequency by altitude diagrams of Yuter and Houze (1995), but replacing height as the ordinate with $\theta_{e}$, show the frequency of updrafts of a given magnitude having the indicated $\theta_{e}$ values. For these diagrams, the bin sizes for vertical velocity and $\theta_{e}$ were $1 \mathrm{~m} \mathrm{~s}^{-1}$ and $2 \mathrm{~K}$, respectively. Frequencies were obtained for an area $160 \times 160 \mathrm{~km}^{2}$ centered on the storm, for heights ranging from $2-6 \mathrm{~km}$, and for the period 62 $66 \mathrm{~h}$ (using output at 2-h intervals). Also shown in the diagrams are the mean values of $\theta_{e}$ for each vertical velocity.

For the Burk-Thompson case (Fig. 11a), the distribution shows that a small number of updrafts of varying magnitudes are associated with $\theta_{e}$ exceeding $360 \mathrm{~K}$. The Blackadar PBL case (Fig. 11c) also produces some updrafts with $\theta_{e}>360 \mathrm{~K}$, but not as many and at somewhat lower values of $\theta_{e}$. Updrafts in the bulk-aerodynamic scheme (Fig. $11 \mathrm{~b}$ ) rarely have $\theta_{e}$ in excess of $358 \mathrm{~K}$, while $\theta_{e}$ in updrafts in the MRF PBL scheme (Fig. 11d) rarely exceed $356 \mathrm{~K}$. The average values of $\theta_{\varepsilon}$ as a function of vertical velocity (thick solid lines) suggest that updrafts in the Burk-Thompson case tend to have the highest $\theta_{e}$ while updrafts in the MRF case have the lowest $\theta_{e}$. The average values in the bulk-aerodynamic and Blackadar PBL cases are about the same. These results indicate that the $\theta_{e}$ fields are generally consistent with the differences in intensity among the PBL cases, with the exception of the difference between the bulkaerodynamic and Blackadar cases. For these cases, one must take into consideration differences in momentum dissipation by friction, as shown below.

\section{b. PBL tendencies}

To diagnose the processes by which the different PBL parameterizations contributed to or inhibited development of the simulated storm, the PBL tendencies for the horizontal velocity 
components, temperature, and water vapor were outputted directly from the model at 2-h intervals (corresponding to the times at which restart files were saved during the simulation). Azimuthally and temporally $(62-66 \mathrm{~h})$ averaged tendencies for the radial and tangential velocities, temperature, and water vapor for each case are shown in Figs. 12-15. Note that the vertical scale in the plots for the MRF case is $2.5 \mathrm{~km}$ compared to $1 \mathrm{~km}$ for the other cases. Also note the contour interval differences for the moisture tendencies between the bulk-aerodynamic PBL case and the other cases.

Qualitatively speaking, the radial and tangential velocity tendencies (Figs. 12-13) in each case are similar. Each case shows the deceleration of both the radial inflow and the vortex circulation. However, the tendencies vary quantitatively in some significant ways. The MRF and Blackadar radial and tangential velocity tendencies are weaker than in the Burk-Thompson case, as should be expected given the weaker winds in these cases. The velocity tendencies in the bulk-aerodynamic case are also much weaker than those in the Burk-Thompson case, despite the fact that the radial and tangential velocities are comparable. This result suggests that, for a hurricane circulation of a given intensity, there is less spin down of the circulation in the bulkaerodynamic case than in the Burk-Thompson case. For a given amount of upward mass transport or diabatic heating, the bulk-aerodynamic case is more efficient at spinning up a strong circulation since less momentum dissipation is being imposed at the surface. This statement appears to explain why the tangential winds in the bulk-aerodynamic case (Fig. 8b) can be as large as in the Burk-Thompson case (Fig. 8a) despite the storm having much weaker vertical motions (Figs. 7a,b).

Temperature (Fig. 14) and moisture (Fig. 15) tendencies in the MRF and bulkaerodynamic PBL cases differ markedly from those in the Blackadar and Burk-Thompson cases. 
The MRF PBL scheme (Figs. 14d, 15d) produces strong drying and warming of the lowest $1 \mathrm{~km}$ and cooling and moistening between about $1-3 \mathrm{~km}$. These strong and deep temperature and moisture tendencies are produced in association with large values of the eddy exchange coefficient that also extend through a deep layer (not shown). The height of the PBL, $h$, in this case averages to about $3 \mathrm{~km}$ in the eye-wall region and results from the equation defining the height of the PBL (Eq. A1). In the case of the hurricane boundary layer, the static stability (the denominator in Al) tends to be relatively small while the winds are strong, resulting in a large value of $h$. This overestimate of $h$ can be reduced by applying the corrections of Vogelezang and Holtslag (1996), in which the shear is taken as the difference in wind speed between the top of the PBL and the top of the surface layer rather than assuming that the wind speed is zero at the bottom of the boundary layer. However, applying this correction in the model leads to little improvement of the simulation (not shown). Another problem is that the PBL scheme does not take into account the effects of clouds. For example, in the eye-wall region, a typically diagnosed value of $h$ is about $3 \mathrm{~km}$, whereas convective cloud bases are generally $\sim 500 \mathrm{~m}$ or less. Hence. the diagnosed PBL top is well above the level at which cumulus transports start to dominate vertical mixing.

The bulk-aerodynamic PBL scheme (Figs. 14b, 15b), which was designed for models with poor vertical resolution, produces temperature and moisture tendencies that are concentrated near the surface and are, in the case of moisture, very strong compared to the other cases. These tendencies are likely not representative of the real atmosphere in high wind environments, and so we might conclude that the bulk-aerodynamic scheme produces the right answer (in terms of intensity and precipitation structure) for the wrong reasons. 
The temperature and water vapor tendencies in the Burk-Thompson (Figs. 14a, 15a) and Blackadar (Figs. 14c, 15c) cases are qualitatively similar. The temperature tendencies for both cases show maximum warming of about the same magnitude below $0.4 \mathrm{~km}$ in the eye-wall region. The Burk-Thompson case produces some weak cooling above $0.4 \mathrm{~km}$. The moisture tendencies show maximum moistening of the boundary layer in the eye-wall region and weaker moistening, peaking near the middle of the boundary layer, in the region outside of the eye wall. Quantitatively, the key difference is in the magnitude of the moisture tendencies in the eye-wall region, with the moistening being much stronger in the Burk-Thompson case. This greater moistening of the PBL in the Burk-Thompson case is an important factor contributing to the higher $\theta_{\ell}$ in the PBL and the greater intensity of the hurricane in this case. Another factor is the frictional dissipation of momentum, which is not obvious in Figs. 12 and 13 because of the different wind speeds of these cases.

It has been shown that the manner in which the vertical mixing is parameterized impacts the storm intensity. The MRF PBL scheme tends to produce excessively deep mixing while the bulk-aerodynamic scheme produces very shallow mixing. An important issue for the Blackadar PBL scheme is the mixing that occurs in the free-convection regime. In this regime, a mixedlayer model is used that assumes that mixing is accomplished by vertically rising convective plumes. However, studies by Moss and Rosenthal (1975), Moss and Merceret (1976), Moss (1978) and Anthes and Chang (1978) have shown that turbulence within the hurricane PBL is dominated by mechanical mixing. If the Blackadar scheme were to diagnose the free-convection regime in an area of strong winds in the hurricane, then the mixing in these regions would be accomplished by the wrong mechanism. However, examination of the PBL regimes diagnosed by the Blackadar scheme indicates that the area of strong winds $\left(>20 \mathrm{~m} \mathrm{~s}^{-1}\right)$ is almost always in 
the forced-convection regime. In this regime, the Blackadar scheme is similar to the bulkaerodynamic PBL scheme in that the parameterizations calculate tendencies of the surface layer variables and use the same formulation for mixing above the lowest model level. However, we have seen in Figs. 14-15 that the bulk-aerodynamic and Blackadar schemes yield very different vertical structures for the PBL tendencies. These differences apparently arise from differences in the surface flux parameterizations and the numerical techniques used for the free-atmospheric mixing above the surface layer (see Appendices A and B).

\section{c. Surface fluxes}

Since theory and idealized modeling of hurricanes indicates a relationship between hurricane intensity and the drag coefficients for heat and momentum, it is instructive to compare the drag coefficients in each scheme. However, since the surface layer characteristics vary in each case, it can be difficult to fairly evaluate the PBL schemes unless identical winds, temperatures, and vapor mixing ratios are used in the calculations. Here, we use simulation results from the Burk-Thompson case at $62 \mathrm{~h}$ to evaluate surface fluxes and drag coefficients for heat, moisture, and momentum $\left(C_{\theta}, C_{q}\right.$, and $C_{. H}$. respectively). See Appendix B for a summary of the surface flux algorithms for each PBL scheme. The MRF and Blackadar PBL schemes use nearly identical surface flux algorithms, so only the Blackadar case is discussed. An important factor to note from Appendix B is the different treatment of the surface roughness parameter, $z_{0}$, in each scheme. In the bulk-aerodynamic scheme, $z_{0}$ is independent of the surface wind speed, whereas in the Blackadar and Burk-Thompson schemes, $z_{0}$ varies linearly with the square of the friction velocity, $u_{*}^{2}$. However, the rate at which $z_{0}$ increases with $u_{*}^{2}$ in the Blackadar case is more than double that in the Burk-Thompson case. 
Table 1 shows area-averaged, minimum, and maximum values of the surface heat, moisture, and momentum fluxes as well as exchange coefficients, obtained from a $280 \times 280 \mathrm{~km}^{2}$ area centered on the storm at $62 \mathrm{~h}$. The Burk-Thompson scheme produces the largest moisture fluxes, while the bulk-aerodynamic and Blackadar schemes are comparable (hence, the comparable values of $\theta_{e}$ within the updrafts in Fig. 11). While the average moisture fluxes differ by only $100 \mathrm{~W} \mathrm{~m}^{-2}$, the maximum values (from the eye-wall region) differ by more than $600 \mathrm{~W}$ $\mathrm{m}^{-2}$. Heat fluxes are significantly less than the moisture fluxes, ranging from a factor of $3-4$ smaller in the Blackadar scheme to almost an order of magnitude smaller in the Burk-Thompson case. Heat fluxes in the eye-wall region in the Blackadar case are nearly twice those in the bulkaerodynamic and Burk-Thompson cases.

For the bulk-aerodynamic and Burk-Thompson schemes, $C_{\theta}=C_{q}$. The exchange coefficient for heat in the bulk-aerodynamic scheme is nearly uniform with a value of about $1.4 \times 10^{-3}$, while in the Burk-Thompson case, $C_{\theta}$ varies with wind speed and ranges from 1 $2.2 \times 10^{-3}$. For the Blackadar scheme, $C_{\theta} \neq C_{\varphi}$. In fact, $C_{\varphi}$ is fairly uniform and small whereas $C_{\theta}$ varies strongly with wind speed and is large. Values of $C_{q}$ range from $1-1.5 \times 10^{-3}$ while $C_{\theta}$ ranges from $1-2.9 \times 10^{-3}$. The differences between $C_{q}$ and $C_{\theta}$ in the Blackadar scheme are due to the term $k u_{*} z_{a} / K_{a}$ in the logarithm in Eq. (B9), which can be several orders of magnitude larger than $z_{a} / z_{0}$. The fact that $C_{q}$ is smaller in the Blackadar case than in the Burk-Thompson case partially accounts for the weaker intensity of the Blackadar case.

Drag coefficients $\left(C_{M}\right)$ are listed in Table 1 and are plotted in Fig. 16 as a function of wind speed along with estimates of $C_{M}$ from Hawkins and Imbembo (1976) in Hurricane Inez (1966). The bulk-aerodynamic drag coefficient is nearly uniform because of the lack of a wind speed dependence of $z_{0}$. If the wind speed dependence of $z_{0}$ were taken into account, Moss and 
Rosenthal's (1975) results suggest that the surface exchange coefficients for heat and momentum derived from the Deardorff (1972) (bulk-aerodynamic PBL) model would agree better with values obtained from budgets within hurricanes. The maximum stress, $\tau_{s}$, in the eye-wall region in the bulk-aerodynamic case is half that in the Burk-Thompson case and almost one-third of that in the Blackadar case. As mentioned previously, the weaker momentum dissipation in the bulkaerodynamic case enables it to produce a strong hurricane despite the relatively weaker surface fluxes of heat and moisture and weaker vertical motions (Fig. 7). The values of $C_{M}$ from the Blackadar scheme agree fairly well with the Hawkins and Imbembo values up to a wind speed of $45 \mathrm{~m} \mathrm{~s}^{-1}$, but are smaller at higher velocities. The Burk-Thompson values of $C_{M}$ parallel the Blackadar values, but are about $30 \%$ less because of the weaker dependence of $z_{0}$ on wind speed (Appendix B). The comparison to the Hawkins and Imbembo data suggests that the $z_{0}$ windspeed dependence in the Burk-Thompson scheme (Eq. B15) should be replaced by that in the Blackadar scheme (Eq. B12).

Emanuel (1995) has derived equations that indicate that the maximum wind and minimum central pressure in hurricanes is proportional to the ratio $C_{h} / C_{M}$, where $C_{h}$ is the drag coefficient for heat. In the bulk-aerodynamic and Burk-Thompson PBL schemes, $C_{h}=C_{\theta}=C_{q}$, whereas in the MRF and Blackadar PBL schemes $C_{\theta} \neq C_{q}$, so in the latter cases we evaluate the ratio using both $C_{\theta}$ and $C_{q}$ separately. Values of $C_{h} / C_{M}$, shown in the last column of Table 1 , are evaluated using the mean values of the drag coefficients rather than the maximum values found in the eye-wall region. Use of the maximum values leads to similar results. The MRF and Blackadar schemes yield $C_{h} / C_{M}$ equal to 1.0 and 0.68 when $C_{\theta}$ and $C_{q}$, respectively, are used for $C_{h}$. Since moisture fluxes provide a greater amount of energy to the PBL, the value of 0.68 is more appropriate for comparison with the other PBL schemes. The Burk-Thompson and bulk- 
aerodynamic PBL schemes are associated with $C_{h} / C_{M}$ values of 1.01 and 1.33 . The much larger values of $C_{h} / C_{M}$ in the bulk-aerodynamic and Burk-Thompson schemes appear to account for the more intense simulations in those cases compared to the MRF and Blackadar cases. The bulk-aerodynamic scheme has a larger value of $C_{h} / C_{M}$ than the Burk-Thompson scheme, but yields a similar central pressure and maximum wind. In contrast, the MRF and Blackadar schemes have identical values of $C_{h} / C_{M}$, but produce different central pressures and maximum winds. These results suggest that the manner in which the vertical mixing is parameterized impacts the storm intensity, but that parameterizations that have larger values of $C_{h} / C_{M}$ will generally be more effective in producing strong hurricanes. Note that increasing $z_{0}$ in the BurkThompson scheme, as suggested above, will not change the value of $C_{h} / C_{M}$ since both coefficients will be increased equally.

\section{Conclusions}

The PSU-NCAR mesoscale model MM5 has been used to simulate Hurricane Bob (1991) at high resolution. The model was able to reproduce fairly realistically the track and intensity of the hurricane, but results exhibited strong sensitivity to the parameterization of boundary layer processes. This study describes this sensitivity and provides detailed analysis of the components of these boundary layer schemes that produce it.

The PBL parameterizations include the Burk-Thompson, MRF, Blackadar, and bulkaerodynamic PBL schemes. Each scheme is different in its formulation of the atmospheric PBL fluxes as well as the surface fluxes, with the exception of the MRF and Blackadar schemes, which share essentially the same surface flux parameterization. Simulated sea-level pressures at storm center varied by about $17 \mathrm{mb}$ among the sensitivity tests, with the Burk-Thompson and 
bulk-aerodynamic schemes producing the strongest storms and the MRF PBL scheme producing the weakest storm. Horizontal precipitation structures also varied substantially, suggesting that accurate forecasts of precipitation in hurricanes can be just as sensitive to the formulation of the PBL as they are to the cloud microphysical parameterization.

The results of the sensitivity tests and diagnoses of the PBL tendencies and surface exchange coefficients are consistent with prior numerical and theoretical studies that suggest that the intensity of hurricane is proportional to the heat input from the ocean and inversely proportional to the frictional dissipation. For example, Emanuel (1995) showed that the minimum central pressure and maximum wind is proportional to $\left(C_{h} / C_{M}\right)^{1 / 2}$, where $C_{h}$ and $C_{M}$ are the surface exchange coefficients for heat and moisture. The simulation results suggest that while the intensity of the storm increases with increasing values of $C_{h} / C_{M}$, the formulation of the atmospheric fluxes above the surface also affects the intensity.

Findings specific to the individual PBL parameterizations include:

- The MRF PBL scheme produced the weakest storm, partly because of a small value of $C_{h} / C_{M}$, but also because the scheme overestimated the depth of the PBL and produced drying in the lower portion of it. The PBL height in this parameterization is calculated from the bulk properties of the boundary layer, and under conditions of weak stability and very strong winds, can reach $2-3 \mathrm{~km}$, which is much greater than observed boundary layer heights within hurricanes. The effect of this overestimate of PBL height was a deep layer of mixing that extended well above cloud base, where vertical mixing by convection usually dominates. This error can be reduced somewhat by using the correction of Vogelezang and Holtslag (1996), but probably also requires using a constraint on the PBL height that accounts for the effects of clouds. 
- The Blackadar PBL scheme produced a weak storm primarily because of weak surface fluxes of moisture relative to those of heat and momentum. The exchange coefficients for heat and moisture differ only by the inclusion of an additional term in the expression for the moisture exchange coefficient, but this additional term produces a significantly reduced dependence of the exchange coefficient on wind speed, and consequently, reduced moisture fluxes at the surface in the interior of the storm. If the exchange coefficient for moisture were set equal to that for heat, then the magnitude of $C_{h} / C_{M}$ would increase from 0.7 to 1 , so that a storm with an intensity comparable to the Burk-Thompson case would be expected.

- The bulk-aerodynamic PBL parameterization produces a very realistic hurricane, but boundary-layer characteristics do not appear to be entirely realistic. The tendencies of temperature and moisture are very shallow, limited primarily to the lowest model level. A major drawback of the scheme is that the surface roughness parameter is independent of the wind speed, which leads to likely underestimates of the surface fluxes of heat, moisture, and momentum in the eye-wall region. However, the large value of $C_{h} / C_{y}$ apparently allows for an intense hurricane to develop.

- The Burk-Thompson PBL scheme produces a reasonably accurate simulation of Hurricane Bob's intensity. The surface drag coefficients in the high-wind regions appear to be underestimated in this scheme because of the formulation of the wind-speed dependence of the surface roughness parameter, $z_{0}=b u_{*}^{2} / g$, with $b=0.0144$. The Blackadar and MRF schemes use $b=0.032$ and produce drag coefficients that are closer to values diagnosed within hurricanes. Substitution of this larger value of $b$ into the Burk-Thompson scheme 
may not significantly change the intensity of the storm, however, since $C_{h} / C_{M}$ will be unchanged.

The Burk-Thompson PBL simulation provides a unique opportunity to examine the structure and evolution of the simulated storm and the processes that contributed to intensification. A series of diagnostic analyses of the model output are underway and results will be presented in future journal publications.

Acknowledgments. The authors gratefully acknowledge Joanne Simpson and Brad Ferrier for their helpful comments, Hugh Willoughby for providing the aircraft and radar data, and Sam Houston for providing the surface wind analysis. Acknowledgment is also made to Dr. R. Kakar (NASA/HQ) for his support of this research and NASA Goddard Space Flight Center for computer time used in the research.

\section{APPENDIX A}

\section{Boundary layer parameterizations}

\section{a. The bulk-aerodynamic scheme}

The bulk-aerodynamic PBL scheme (Deardorff 1972; Grell et al. 1995) treats the first full- $\sigma$ model level ( $86 \mathrm{~m}$ in this case) as the top of the boundary layer and uses similarity theory to determine surface fluxes of momentum, heat, and moisture, and tendencies of these variables at the first half- $\sigma$ model level $(42 \mathrm{~m}$ ). Above the first level, $K$-theory is used for mixing in the free atmosphere. Tendencies of the model variables are then calculated from $\partial C /\left.\partial t\right|_{P B L}=\partial / \partial z\left(K_{C} \partial C / \partial z\right)$, where $C \in\left(u, v, \theta, q_{v}\right)$, using centered finite differences. The eddy 
diffusivity coefficient, $K_{C}$, is specified as a function of the local Richardson number following Blackadar (1976) and Zhang and Anthes (1982).

\section{b. The Blackadar PBL scheme}

The Blackadar PBL scheme (Balckadar 1976, 1979; Zhang and Anthes 1982; Oncley and Dudhia 1995) contains two different regimes of turbulent mixing: a stable, or nocturnal, regime and a free-convection regime. The stable regime is divided into three categories with the appropriate category being determined by the sign and magnitude of the bulk Richardson number, Rib. When $\mathrm{Rib} \geq 0.2$, the surface is assumed to be very stable, while for $0<\mathrm{Rib}<0.2$ the surface layer is assumed to be in a state of damped mechanical turbulence. When Rib $\leq 0$ and $\left|z_{u} / L\right| \leq 1.5$ ( $z_{a}$ is the height of the first half- $\sigma$ level, $L$ is the Monin-Obukhov length scale), a state of forced convection is assumed. Tendencies of surface variables are determined from a local- $K$ approach. Above the surface layer, mixing is determined from $K$-theory in the same manner as in the bulk-aerodynamic scheme. Basically, the nocturnal regime is similar to the bulk-aerodynamic PBL parameterization, but the Blackadar scheme uses a different formulation of the surface fluxes (Appendix B) and uses the implicit diffusion technique of Richtmyer (1957. Ch. 6) instead of centered finite differences to compute the mixing above the surface layer.

In the free-convection regime, Rib $\leq 0$ and $\left|z_{a} / L\right| \geq 1.5$, the vertical transfers of heat, moisture, and momentum are not determined by local gradients, but by the thermal structure of the whole mixed layer and the surface heat flux. Prognostic variables within the mixed layer are modified by assuming that vertical exchanges take place between the lowest layer and each level of the mixed layer. See Zhang and Anthes (1982) for details of the free-convection mixing scheme. Above the mixed layer, mixing is determined from $K$-theory as before. 


\section{c. The MRF PBL scheme}

The MRF scheme (Hong and Pan, 1996) is a nonlocal scheme in which the tendencies are dependent on the bulk characteristics of the PBL and include counter-gradient transports of temperature and moisture that account for the contributions from large-scale eddies (the countergradient term for moisture is set to zero over the ocean in the MM5 code). The eddy diffusivity coefficient for momentum, $K_{m}$, is a function of the friction velocity, $u_{*}$, and the PBL height, $h$, given by

$$
h=\operatorname{Rib}_{\mathrm{cr}} \frac{\theta_{\mathrm{va}}|V(h)|^{2}}{g\left[\theta_{v}(h)-\theta_{s}\right]}
$$

where $\mathrm{Rib}_{\mathrm{cr}}$ is the critical bulk Richardson number $(=0.5), V(h)$ and $\theta_{v}(h)$ are the wind speed and virtual potential temperature at the top of the PBL, $\theta_{s}=\theta_{v a}+\theta_{T}$ is a near surface potential temperature given in Eq. (9) of Hong and Pan (1996), $\theta_{i: u}$ is the virtual potential temperature at the lowest half- $\sigma$ level, and $\theta_{T}$ is a scaled virtual temperature excess near the surface that incorporates the effects of surface heat fluxes. The eddy diffusivity for temperature and moisture is computed from $K_{m}$ by using a Prandtl number relationship given in Eq. (10) of Hong and Pan (1996).

\section{d. The Burk-Thompson scheme}

The Burk-Thompson PBL scheme (Burk and Thompson 1989) is a Mellor-Yamada level2.5 closure model that includes a prognostic equation for the turbulent kinetic energy (TKE). In contrast to the description of the level-3 equations in Burk and Thompson (1989), the scheme in MM5 neglects the effects of liquid water as well as the counter-gradient terms in the fluxes of 
heat and moisture. The eddy diffusivity for moisture is taken as $K_{q}=1.0075 K_{h}\left(K_{h}\right.$ being the eddy exchange coefficient for heat) rather than as a function of the vertical velocity variance. The fluxes are derived from a local- $K$ approach, but unlike the free-atmosphere formulations for eddy diffusivity in the Blackadar and bulk-aerodynamic scheme, in which the eddy diffusivity is a function of the local Richardson number, the eddy diffusivity in the Burk-Thompson scheme is given by a complex algebraic function involving the predicted mean and turbulence variables.

\section{APPENDIX B}

\section{Surface flux parameterizations}

The parameterizations of the surface fluxes of heat, moisture and momentum play a key role in the simulation of hurricanes, which gain energy through transfer of sensible and latent heat at the surface and lose energy to frictional dissipation. In the following subsections, we briefly summarize the surface flux algorithms used in each PBL scheme. For this discussion, we use a generic framework in which the surface fluxes of heat $\left(H_{s}\right)$, moisture $\left(E_{s}\right)$, and momentum $\left(\tau_{s}\right)$ are given by

$$
\begin{gathered}
H_{s}=\rho_{a} c_{p} C_{\theta} V_{a}\left(\theta_{g}-\theta_{a}\right) \\
E_{s}=\rho_{a} L_{v} M C_{q} V_{a}\left[q_{v s}\left(T_{g}\right)-q_{v a}\right] \\
\tau_{s}=\rho_{a} C_{M} V_{a}^{2}
\end{gathered}
$$

where $\rho_{a}, q_{v a}$, and $V_{a}$ are the air density, vapor mixing ratio, and velocity at the top of the surface layer; $q_{v s}$ is the saturation vapor mixing ratio at the surface and is a function of the sea-surface temperature $T_{g} ; C_{\theta}, C_{q}$, and $C_{M}$ are exchange coefficients for heat, moisture, and momentum, 
respectively; $L_{v}$ is the latent heat of vaporization; and $M$ is the moisture availability ( $M=1$ over the ocean). The differences between the different PBL schemes is then contained in the three exchange coefficients $C_{\theta}, C_{q}$, and $C_{M}$. For this discussion, only exchange coefficients for unstable conditions are presented.

\section{a. The bulk-aerodynamic scheme}

Exchange coefficients in the bulk-aerodynamic scheme are defined following Deardorff (1972), with $C_{M}=C_{u}^{2}$ and $C_{\theta}=C_{q}=C_{T} C_{u}$. For unstable conditions $(\mathrm{Rib}<0)$,

$$
\begin{gathered}
C_{u}=\left[\frac{1}{C_{u N}}-25 \exp \left(0.26 \varphi-0.03 \varphi^{2}\right)\right]^{-1} \\
C_{T}=\left[\frac{1}{C_{T N}}+\frac{1}{C_{u}}-\frac{1}{C_{u N}}\right]^{-1}
\end{gathered}
$$

where Ric=3.05, $\varphi=\log _{10}(-\mathrm{Rib})-3.5, \mathrm{Rib}=\left(g h / \theta_{0}\right)\left(\theta_{a}-\theta_{g}\right) / V^{2}, h$ is the height of the first full $-\sigma$ level, $\theta_{0}=283.16, \theta_{u}$ and $\theta_{g}$ are the potential temperatures at the first half- $\sigma$ level and the ground, and $V$ is a combination of the wind speed and a convective velocity (see Grell et al. 1995). The velocity $V$ is used in (B1-B3) instead of the actual wind speed $V_{u}$. The parameters $C_{u k}$ and $C_{T V}$ are the neutral values for $C_{u}$ and $C_{T}$ given by

$$
\begin{gathered}
C_{u, N}=\left[\frac{1}{k} \ln \left(\frac{z_{a}}{z_{0}}\right)+8.4\right]^{-1} \\
C_{r N}=\left[\frac{0.74}{k} \ln \left(\frac{z_{u}}{z_{0}}\right)+7.3\right]^{-1}
\end{gathered}
$$


where $z_{a}=0.025 h, z_{0}$ is the surface roughness parameter, and $k$ the von Karman constant $(k=0.4)$. It is important to note that, unlike the other three PBL schemes, in the bulk-aerodynamic PBL scheme, $z_{0}$ is independent of the wind speed over the ocean. Also, unlike the other schemes that take $z_{a}$ as the height of the first half- $\sigma$ level, the bulk scheme assumes that the surface layer depth is a small fraction of the height of the first full- $\sigma$ level.

\section{b. The MRF and Blackadar PBL schemes}

The MRF and Blackadar schemes use nearly identical representations of the surface fluxes. For these schemes, $C_{\theta} \neq C_{4}$, and the exchange coefficients can be expressed as follows

$$
\begin{gathered}
C_{\theta}=k^{2}\left(\ln \frac{z_{a}}{z_{0}}-\varphi_{m}\right)^{-1}\left(\ln \frac{z_{a}}{z_{0}}-\varphi_{h}\right)^{-1} \\
C_{q}=k^{2}\left(\ln \frac{z_{a}}{z_{0}}-\varphi_{m}\right)^{-1}\left[\ln \left(\frac{k u_{*} z_{a}}{K_{a}}+\frac{z_{a}}{z_{0}}\right)-\varphi_{h}\right]^{-1} \\
C_{M}=u_{*}^{2} / V^{2}
\end{gathered}
$$

where $K_{a}$ is a background molecular diffusivity $\left(=2.4 \times 10^{-5} \mathrm{~m}^{2} \mathrm{~s}^{-1}\right), u_{*}$ is the friction velocity

$$
u_{*}=\operatorname{MAX}\left[\frac{k V}{\ln z_{a} / z_{0}-\varphi_{m}}, u_{* 0}\right] \text {, }
$$

$z_{u}$ is the height of the first half- $\sigma$ level, and $\varphi_{m}$ and $\varphi_{h}$ are nondimensional stability functions that depend on the PBL regime (i.e., stable, mechanical turbulence, forced or free convection). In 
Eq. (B9), the first term in the second logarithm can be rewritten as $z_{a} / z_{k}$, with $z_{k}=K_{a} / k u_{*}$, and is included to add increased resistance to the transfer of water vapor from the surface (Oncley and Dudhia 1995). The maximum value of $z_{k}$ is $0.06 \mathrm{~cm}$, whereas $z_{0}$ can reach $5-10 \mathrm{~cm}$. Consequently, $z_{a} / z_{k}>z_{a} / z_{0}$ and the exchange coefficient for water vapor is smaller than that for heat. The surface roughness parameter, $z_{0}$, varies with wind speed over the ocean, and is prescribed following Delsol et al. (1971),

$$
z_{0}=0.032 u_{*}^{2} / g+z_{0 c}
$$

where $z_{0 c}$ is a background value of $10^{-4} \mathrm{~m}$. As in the bulk-aerodynamic scheme, the velocity scale $V$ is used in (B1-B3) instead of the wind speed $V_{a}$. The primary difference between the Blackadar and MRF PBL schemes is in the definition of the nondimensional stability functions for the free convection regime. For the purposes of this study, this difference is negligible.

c. The Burk-Thompson scheme

For the Burk-Thompson scheme, the surface fluxes are based on Louis $(1979,1982)$, with $C_{\theta}=C_{q}$ and the wind speed $V_{a}$ used in (B1-B3). In the unstable case, Rib $<0$, where $\operatorname{Rib}=\left(g z_{a} / \bar{\theta}_{v}\right)\left(\theta_{v a}-\theta_{v g}\right) / V_{a}^{2}$ and $\bar{\theta}_{v}=\left(\theta_{v a}+\theta_{v g}\right) / 2$, the exchange coefficients are

$$
\begin{aligned}
& C_{\theta}=C_{N}\left[1-\frac{3 b \mathrm{Rib}}{1+3 b c C_{N}\left(-z_{a} \mathrm{Rib} / z_{0}\right)^{1 / 2}}\right] \\
& C_{M}=C_{N}\left[1-\frac{2 b \mathrm{Rib}}{1+3 b c C_{N}\left(-z_{a} \mathrm{Rib} / z_{0}\right)^{1 / 2}}\right]
\end{aligned}
$$


where $b$ and $c$ are constants $\left(b=c=5\right.$ ). The parameter $C_{N}$ is the exchange coefficient for both heat and momentum under neutral conditions, $C_{N}^{1 / 2}=k / \ln \left(z_{a} / z_{0}\right)$, where $z_{a}$ is the height of the first half- $\sigma$ level. As in the MRF and Blackadar schemes, $z_{0}$ is allowed to vary with the wind speed, but in the Burk-Thompson PBL scheme, the relationship follows Garratt (1977),

$$
z_{0}=0.0144 u_{*}^{2} / g
$$

Thus, for the same $u_{*}$, the Burk-Thompson scheme yields a smaller $z_{0}$ than in the MRF and Blackadar schemes. Using (B12) in place of (B15) would increase the drag coefficients, but would not change the value of $C_{\theta} / C_{M}$.

\section{REFERENCES}

Anthes, R. A., and S. W. Chang, 1978: Response of the hurricane boundary layer to changes of sea surface temperature in a numerical model. J. Atmos. Sci., 35, 1240-1255.

Black, R. A., H. B. Bluestein, and M. L. Black, 1994: Unusually strong vertical motions in a Caribbean hurricane. Mon. Wea. Rev., 122, 2722-2739.

Blackadar, A. K., 1976: Modelng the nocturnal boundary layer. Preprints, Third Symp. on Atmospheric Turbulence, Diffusion, and Air Quality, Raleigh, Amer. Meteor. Soc., 46-49.

—_, 1979: High resolution models of the planetary boundary layer. Advances in Environmental Science and Engineering, Vol. 1, No. 1, J. Pfafflin and E. Ziegler, Eds., Gordon and Breach, 50-85. 
Braun, S. A., B. S. Ferrier, and W.-K. Tao, 1999: Parameterization of depositional growth of cloud ice in a bulk microphysical scheme. J. Atmos. Sci., (submitted).

Burk, S. D., and W. T. Thompson, 1989: A vertically nested regional numerical weather prediction model with second-order closure physics. Mon. Wea. Rev., 117, 2305-2324.

Byers, H. R., 1944: General Meteorology. McGraw-Hill Book Co., Inc., New York, NY, 645 pp.

Deardorff, J. W., 1972: Parameterization of the planetary boundary layer for use in general circulation models. Mon. Wea. Rev., 100, 93-106.

Delsol, F., K. Miyakoda, and R. H. Clarke, 1971: Parameterized processes in the surface boundary layer of an atmospheric circulation model. Quart. J. Roy. Meteor. Soc., 97, 181208.

Dudhia, J, 1989: Numerical study of convection observed during the winter monsoon experiments using a mesoscale two-dimensional model. J. Atmos. Sci., 46, 3077-3107.

1993: A nonhydrostatic version of the Penn State-NCAR mesoscale model: Validation tests and simulation of an Atlantic Cyclone and cold front. Mon. Wea. Rev., 121, 1493-1513.

Emanuel, K. A., 1986: An air-sea interaction theory for tropical cyclones. Part I: Steady-state maintenance. J. Atmos. Sci., 43, 585-604.

1995: Sensitivity of tropical cyclones to surface exchange coefficients and a revised steady-state model incorporating eye dynamics. J. Atmos. Sci., 52, 3969-3976.

1997: Some aspects of hurricane inner-core dynamics and energetics. J. Atmos. Sci., 54, $1014-1026$.

Ferrier, B. S., W.-K. Tao, and J. Simpson, 1995: A double-moment multiple-phase four-class bulk ice scheme. Part II: Simulations of convective storms in different large-scale 
environments and comparisons with other bulk parameterizations. J. Atmos. Sci., 52, 1001 1033.

Garratt, J. R., 1977: Review of drag coefficients over oceans and continents. Mon. Wea. Rev., 105, 915-929.

Grell, G. A., J. Dudhia, and D. R. Stauffer, 1995: A description of the fifth-generation Penn State/NCAR Mesoscale Model (MM5). NCAR Technical Note (NCAR/TN-398+STR), 122 pp.

Hawkins, H. F., and S. M. Imbembo, 1976: The structure of a small, intense hurricane-Inez 1966. Mon. Wea. Rev., 104, 418-442.

Holland, G. J., 1997: The maximum potential intensity of tropical cyclones. J. Atmos. Sci., 54, 2519-2541.

Hong, , S.-H., and H.-L. Pan, 1996: Nonlocal boundary layer vertical diffusion in a mediumrange forecast model. Mon. Wea. Rev., 124, 2322-2339.

Houston, S. H., and M. D. Powell, 1993: Surface wind fields during Hurricane Bob's (1991) landfall in New England. Preprints, $20^{\text {th }}$ Conf. on Hurricanes and Tropical Meteorology, San Antonio, TX, Amer. Meteor. Soc., 139-142.

Jorgensen, D. P., 1984: Mesoscale and convective-scale characteristics of mature hurricanes. Part II: Inner core structure of Hurricane Allen (1980). J. Atmos. Sci., 41, 1287-1311.

Karyampudi, V. M., G. S. Lai, and J. Manobianco, 1998: Impact of initial conditions, rainfall assimilation, and cumulus parameterization on simulations of Hurricane Florence (1988). Mon. Wea. Rev., 126, 3077-3101.

Liu, Y., D.-L. Zhang, and M. K. Yau, 1997: A multiscale numerical study of Hurricane Andrew (1992). Part I: Explicit simulation and verification. Mon. Wea. Rev., 125, 3073-3093. 
Lord. S. J., H. E. Willoughby, and J. M. Piotrowicz, 1984: Role of a parameterized ice-phase microphysics in an axisymmetric nonhydrostatic tropical cyclone model. J. Atmos. Sci., 41. 2836-2848.

Louis, J. F., 1979: A parametric model of vertical eddy fluxes in the atmosphere. BoundaryLayer Meteor., 17, 187-202.

$\longrightarrow$, 1982: Workshop on planetary boundary layer parameterization. ECMWF Report, 260 pp. (Available from ECMWF, Shinfield Park, Reading, Berkshire RG2 9AX, United Kingdom).

Malkus, J. S., and H. Riehl, 1960: On the dynamics and energy transformations in steady-state hurricanes. Tellus, 12, 1-20.

Manning, K. W., and P. L. Haagenson, 1992: Data ingest and objective analysis for the PSU/NCAR modeling system: Programs DATAGRID and RAWINS. NCAR Technical Note (NCAR/TN-376+IA), 209 pp.

Moss, M. S., 1978: Low-level turbulence structure in the vicinity of a hurricane. Mon. Wea. Rev.. 106, 841-849. and S. L. Rosenthal, 1975: On the estimation of planetary boundary layer variables in mature hurricanes. Mon. Wea. Rev., 103, 980-988.

$\longrightarrow$, and F. J. Merceret, 1976: A note on several low-layer features of Hurricane Eloise (1975). Mon. Wea. Rev., 104, 967-971.

Meyers, M. P., P. J. DeMott, and W. R. Cotton, 1992: New primary ice-nucleation parameterizations in an explicit cloud model. J. Appl. Meteor., 31, 708-721.

Ooyama, K., 1969: Numerical simulation of the life cycle of tropical cyclones. J. Atmos. Sci., 26, $3-40$. 
Oncley, S. P., and J. Dudhia, 1995: Evaluation of surface fluxes from MM5 using observations. Mon. Wea. Rev., 123, 3344-3357.

Palmén, E., and H. Riehl, 1957: Budget of angular momentum and energy in tropical cyclones. $J$. Meteor., 14, 150-159.

Richtmyer, R. D., 1957: Difference Methods for Initial-Value Problems. New York, Interscience, $238 \mathrm{pp}$.

Riehl, H., 1954: Tropical Meteorology. New York, McGraw-Hill, 392 pp.

Rosenthal, S. L., 1971: The response of a tropical cyclone model to variations in boundary layer parameters, initial conditions, lateral boundary conditions, and domain size. Mon. Wea. Rev., 99. 767-777.

Rotunno, R., and K. A. Emanuel, 1987: An air-sea interaction theory for tropical cyclones. Part II: Evolutionary study using a nonhydrostatic axisymmetric numerical model. J. Atmos. Sci., 44, 542-561.

Rutledge, S. A., and P. V. Hobbs, 1984: The mesoscale and microscale structure and organization of clouds and precipitation in midlatitude cyclones. XII: A diagnostic modeling study of precipitation development in narrow cold-frontal rainbands. J. Atmos. Sci., 41, 29492972.

Tao, W.-K., and J. Simpson, 1993: The Goddard Cumulus Ensemble Model. Part I: Model description. Terrestrial, Atmospheric, and Oceanic Sciences, 4, 35-72.

Vogelezang, D. H. P., and A. A. M. Holtslag, 1996: Evaluation and model impacts of alternative boundary-layer height formulations. Boundary-Layer Meteor., 81, 245-269. 
Yuter, S. E., and R. A. Houze, Jr., 1995: Three-dimensional kinematic and microphysical evolution of Florida cumulonimbus. Part II: Frequency distributions of vertical velocity, reflectivity, and differential reflectivity. Mon. Wea. Rev., 123, 1941-1963.

Zhang, D., and R. A. Anthes, 1982: A high-resolution model of the planetary boundary layer-Sensitivity tests and comparisons with SESAME-79 data. J. Appl. Meteor., 21, 15941609. 


\section{FIGURE CAPTIONS}

Figure 1. Map of the course and fine mesh domains. The first inner box corresponds to the 12 $\mathrm{km}$ domain and the second box to the 4-km domain. Also shown on the map and in the inset in the lower-left corner are the tracks of the observed storm (solid line) and the simulated storm (dashed line) from the 36-km grid simulation. Dots along the tracks are drawn every $6 \mathrm{~h}$ starting at 0000 UTC 16 August 1991.

Figure 2. Comparison of the observed central pressure with values from the $36-\mathrm{km}$ coarse-grid simulation and from the high-resolution simulation with the Burk-Thompson PBL physics (48$72 \mathrm{~h})$.

Figure 3. (a) Radar reflectivities from the Cape Hatteras, NC, NEXRAD radar at 0056 UTC 19 August (courtesy of $\mathrm{H}$. Willoughby, NOAA/HRD). Tick marks are drawn every $40 \mathrm{~km}$. (b) Simulated radar reflectivity pattern at $1 \mathrm{~km} \mathrm{MSL}$ at $t=72 \mathrm{~h}$ (valid at 0000 UTC 19 August) for the high-resolution simulation with the Burk-Thompson PBL. Major tick marks drawn every 40 $\mathrm{km}$. Dashed lines indicate latitude and longitude every $1^{\circ}$.

Figure 4. (a) Surface wind analysis for Hurricane Bob at 0300 UTC 19 August (courtesy of S. Houston, NOAA/HRD). Contours are isotachs drawn every $5 \mathrm{~m} \mathrm{~s}^{-1}$. Shaded region encloses winds exceeding $35 \mathrm{~m} \mathrm{~s}^{-1}$. (b) Simulated winds at the lowest model level (42 $\mathrm{m}$ ) at $t=72 \mathrm{~h}$ valid at 0000 UTC 19 August. Contours are isotachs at $5 \mathrm{~m} \mathrm{~s}^{-1}$ intervals. Thick solid lines correspond to wind speeds of 35 and $50 \mathrm{~m} \mathrm{~s}^{-1}$. 
Figure 5. Time series of central pressure for the $24-\mathrm{h}$ period of the high-resolution simulations. Comparison of the observed central pressure with values from the high-resolution simulations with variable PBL physics.

Figure 6. Simulated radar reflectivity patterns at $1 \mathrm{~km}$ MSL and sea-level pressure at $t=72 \mathrm{~h}$ (valid at 0000 UTC 19 August) for the high-resolution simulations with variable PBL physics. Sea-level pressure contours are drawn every $4 \mathrm{mb}$.

Figure 7. Vertical cross sections of azimuthally and temporally averaged vertical velocity averaged over hours 60-66, using output at 15-min intervals. Positive values are contoured at intervals of $0.15 \mathrm{~m} \mathrm{~s}^{-1}$ (contour labels are in $\mathrm{cm} \mathrm{s}^{-1}$ ), negative values at intervals of $0.05 \mathrm{~m} \mathrm{~s}^{-1}$. Panels correspond to the following PBL schemes: (a) Burk-Thompson, (b) bulk-aerodynamic, (c) Blackadar, and (d) MRF.

Figure 8. Same as in Fig. 7, but for tangential velocity. The contour interval is $5 \mathrm{~m} \mathrm{~s}^{-1}$.

Figure 9. Same as in Fig. 7, but for radial velocity. Positive values are contoured at intervals of 2 $\mathrm{m} \mathrm{s}^{-1}$, negative values at intervals of $4 \mathrm{~m} \mathrm{~s}^{-1}$.

Figure 10. Same as in Fig. 7, but for equivalent potential temperature and cloud water. Contours for $\theta_{\mathrm{e}}$ are drawn at $2 \mathrm{~K}$ intervals. Cloud water amounts are indicated by the stippling, with light and dark stippling indicating values greater than 0.05 and $0.25 \mathrm{~g} \mathrm{~kg}^{-1}$, respectively. 
Figure 11. Contoured frequency diagrams showing the frequency of a given $\theta_{\mathrm{e}}$ value occurring with a given vertical velocity, $w$. Contours show the number of grid points with the given $w$ and $\theta_{\mathrm{e}}$ and are drawn at values of $1,3,6,20,40,60,80,150,200,300,400,750$, and 1000 . The thick solid line indicates the average $\theta_{\mathrm{e}}$ for a given $w$. Panels correspond to the following PBL schemes: (a) Burk-Thompson, (b) bulk-aerodynamic, (c) Blackadar, and (d) MRF.

Figure 12. Vertical cross sections of azimuthally and temporally averaged radial velocity tendency averaged over hours 62-66, using output at 2-h intervals. Contours are drawn at $0.4 \mathrm{~m}$ $\mathrm{s}^{-2}$ intervals. Panels correspond to the following PBL schemes: (a) Burk-Thompson, (b) bulkaerodynamic, (c) Blackadar, and (d) MRF. Note that the vertical scale in (d) is $2.5 \mathrm{~km}$ compared to $1 \mathrm{~km}$ in the other panels.

Figure 13. Same as in Fig. 12, but for tangential velocity tendency. Contours are drawn at $0.15 \mathrm{~m}$ $\mathrm{s}^{-2}$ intervals.

Figure 14. Same as in Fig. 12, but for temperature tendency. Contours are drawn at $1 \mathrm{~K} \mathrm{~h}^{-1}$ intervals.

Figure 15. Same as in Fig. 12, but for temperature tendency. Contours are drawn at $0.5 \mathrm{~g} \mathrm{~kg}^{-1} \mathrm{~h}^{-1}$ intervals in (a, c, and d) and at $3 \mathrm{~g} \mathrm{~kg}^{-1} \mathrm{~h}^{-1}$ intervals in (b).

Figure 16. Exchange coefficients for momentum as a function of horizontal wind speed. 
Table 1. Surface fluxes and exchange coefficients for moisture, heat and momentum at $t=62 \mathrm{~h}$ (see Appendix B for notation). For each PBL scheme, the first number in the column is the average value over a $280 \times 280 \mathrm{~km}^{2}$ area centered on the storm, while the second and third numbers are the minimum and maximum values. In the last column, values of $C_{H} / C_{M}$ for the Blackadar scheme using $C_{h}=C_{\theta}$ and $C_{h}=C_{q}$ are provided.

\begin{tabular}{|c|c|c|c|c|c|c|c|}
\hline Case & $\begin{array}{c}E_{s} \\
\left(\mathrm{~W} \mathrm{~m}^{-2}\right)\end{array}$ & $\begin{array}{c}H_{s} \\
\left(\mathrm{~W} \mathrm{~m}^{-2}\right)\end{array}$ & $\begin{array}{c}\tau_{\mathrm{s}} \\
\left(\mathrm{kg} \mathrm{m}^{-1} \mathrm{~s}^{-2}\right)\end{array}$ & $\begin{array}{c}C_{M} \\
\left(\times 10^{-3}\right)\end{array}$ & $\begin{array}{c}C_{\theta} \\
\left(\times 10^{-3}\right)\end{array}$ & $\begin{array}{c}C_{q} \\
\left(\times 10^{-3}\right)\end{array}$ & $C_{h} / C_{M}$ \\
\hline \multirow{2}{*}{ Burk- } & 686 & 46 & 1.34 & 1.53 & 1.54 & 1.54 & 1.01 \\
Thompson & 36 & -23 & 0.01 & 1.01 & 1.02 & 1.02 & \\
& 2031 & 280 & 6.61 & 2.23 & 2.25 & 2.25 & \\
\hline \multirow{3}{*}{ Bulk } & 615 & 80 & 0.82 & 1.04 & 1.38 & 1.38 & 1.33 \\
& 62 & 6 & 0.017 & 0.99 & 1.30 & 1.30 & \\
& 1322 & 263 & 3.12 & 1.27 & 1.74 & 1.74 & \\
\hline \multirow{3}{*}{ Blackadar } & 585 & 119 & 1.67 & 1.88 & 1.88 & 1.28 & $1.0\left(C_{\theta}\right)$ \\
& 37 & 4 & 0.015 & 1.01 & 1.01 & 0.99 & $0.68\left(C_{q}\right)$ \\
& 1446 & 536 & 8.60 & 2.91 & 2.91 & 1.49 & \\
\hline
\end{tabular}

The PSU-NCAR mesoscale model MM5 has been used to simulate Hurricane Bob (1991) at high resolution. The model was able to reproduce fairly realistically the track and intensity of the hurricane when the Burk-Thompson PBL parameterization was used. Some differences between the simulation and observations included stronger than observed winds near the top of the PBL, a larger radius for the eye wall, and a much smaller area of precipitation and hurricane force winds. However, despite these shortcomings, the simulation should be adequate for examining the processes that contribute to intensification. 


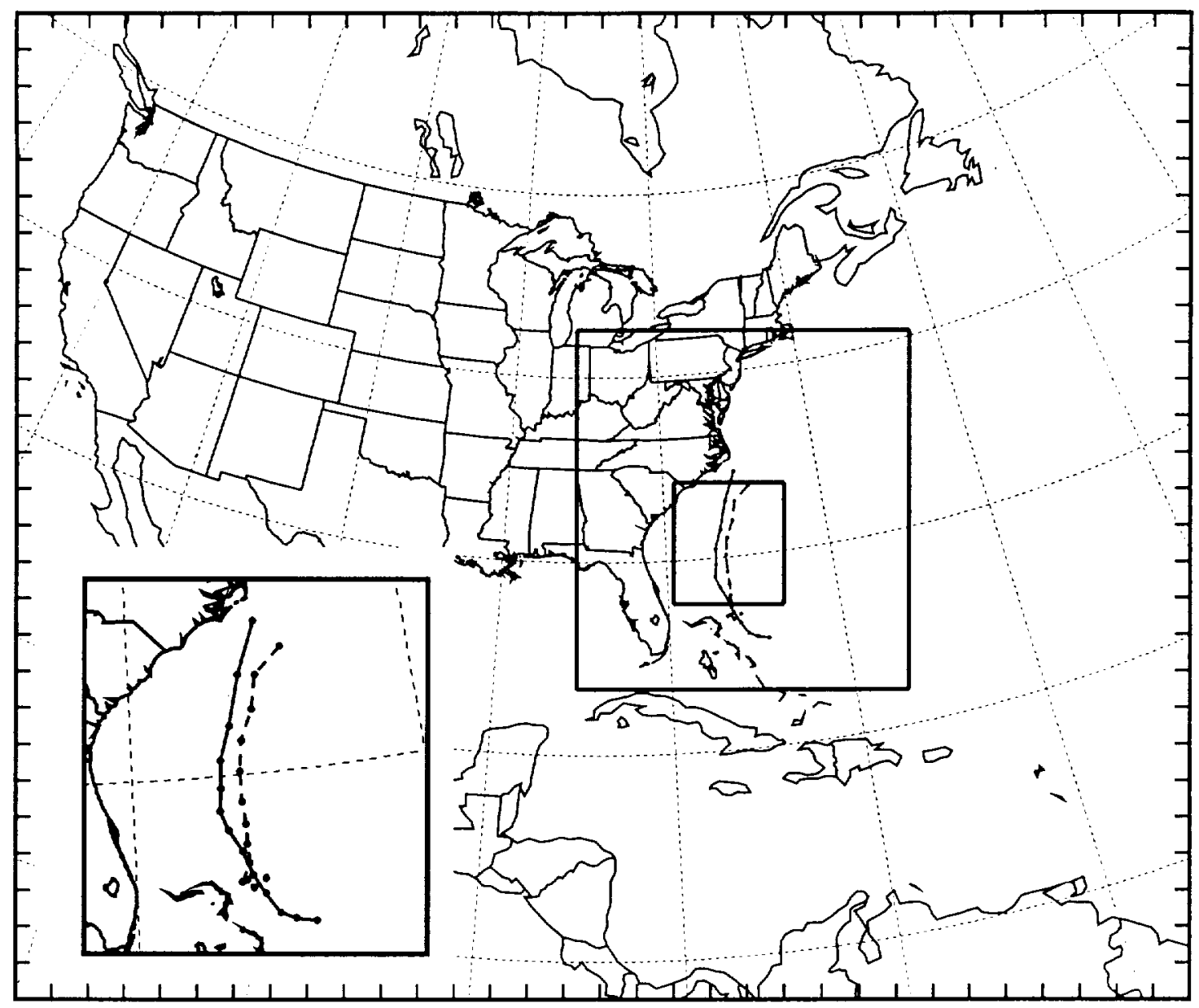

Figure 1. Map of the course and fine mesh domains. The first inner box corresponds to the $12-\mathrm{km}$ domain and the second box to the 4-km domain. Also shown on the map and in the inset in the lower-left corner are the tracks of the observed storm (solid line) and the simulated storm from the $36-\mathrm{km}$ grid simulation. Dots along the tracks are drawn every $6 \mathrm{~h}$ starting at 0000 UTC 16 AUG 1991. 


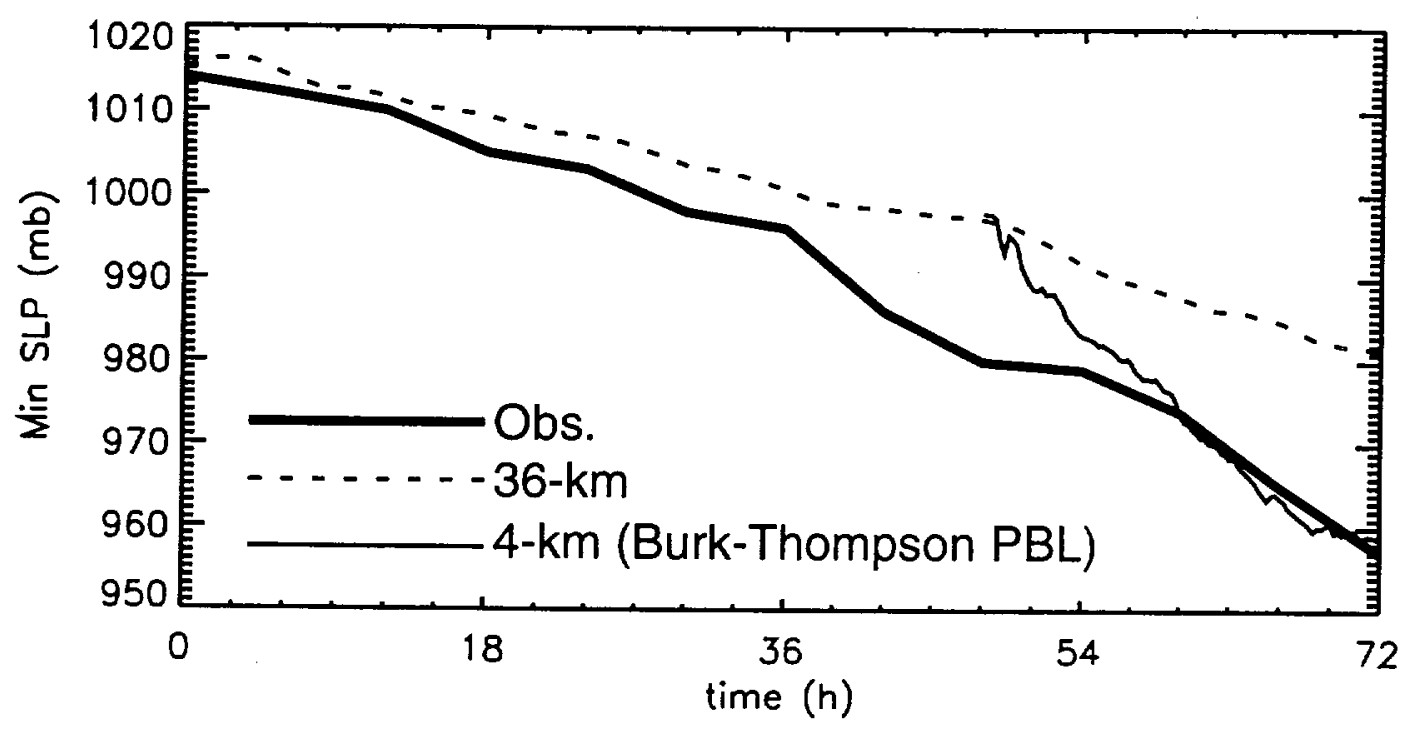

Figure 2. Comparison of the observed central pressure with values from the $36-\mathrm{km}$ coarse grid simulation and from the high-resolution simulation with the Burk-Thompson PBL physics (48-72 h). 

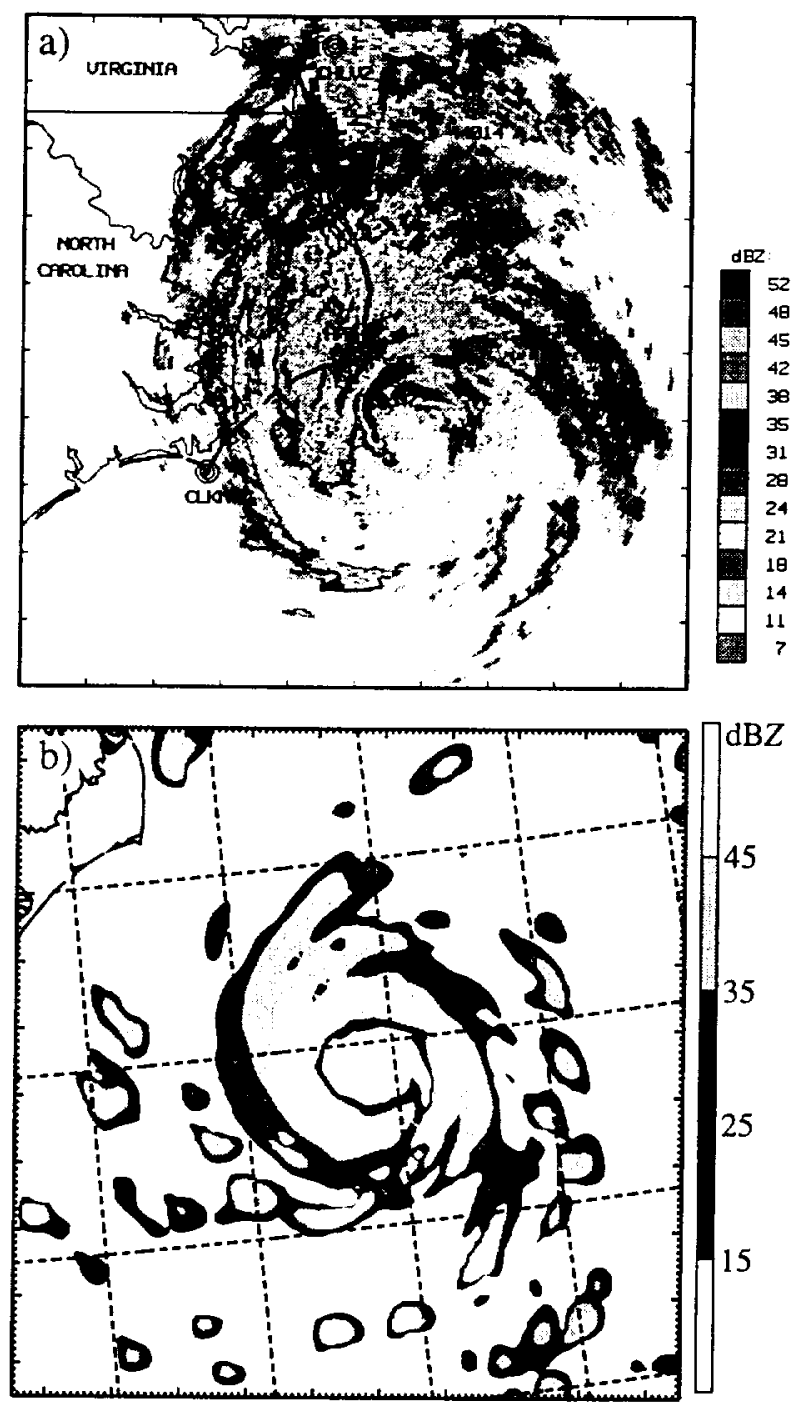

Figure 3. (a) Radar reflectivities from the Cape Hatteras, NC, NEXRAD radar at 0056 UTC 19 August (courtesy of H. Willoughby, NOAA/HRD). Tick marks are drawn every $40 \mathrm{~km}$. (b) Simulated radar reflectivity pattern at $1 \mathrm{~km} \mathrm{MSL}$ at $t=72 \mathrm{~h}$ (valid at 0000 UTC 19 August) for the high-resolution simulation with the Burk-Thompson PBL. Major tick marks are drawn every $40 \mathrm{~km}$. Dashed lines indicate latitude and longitude every $1^{\circ}$. 

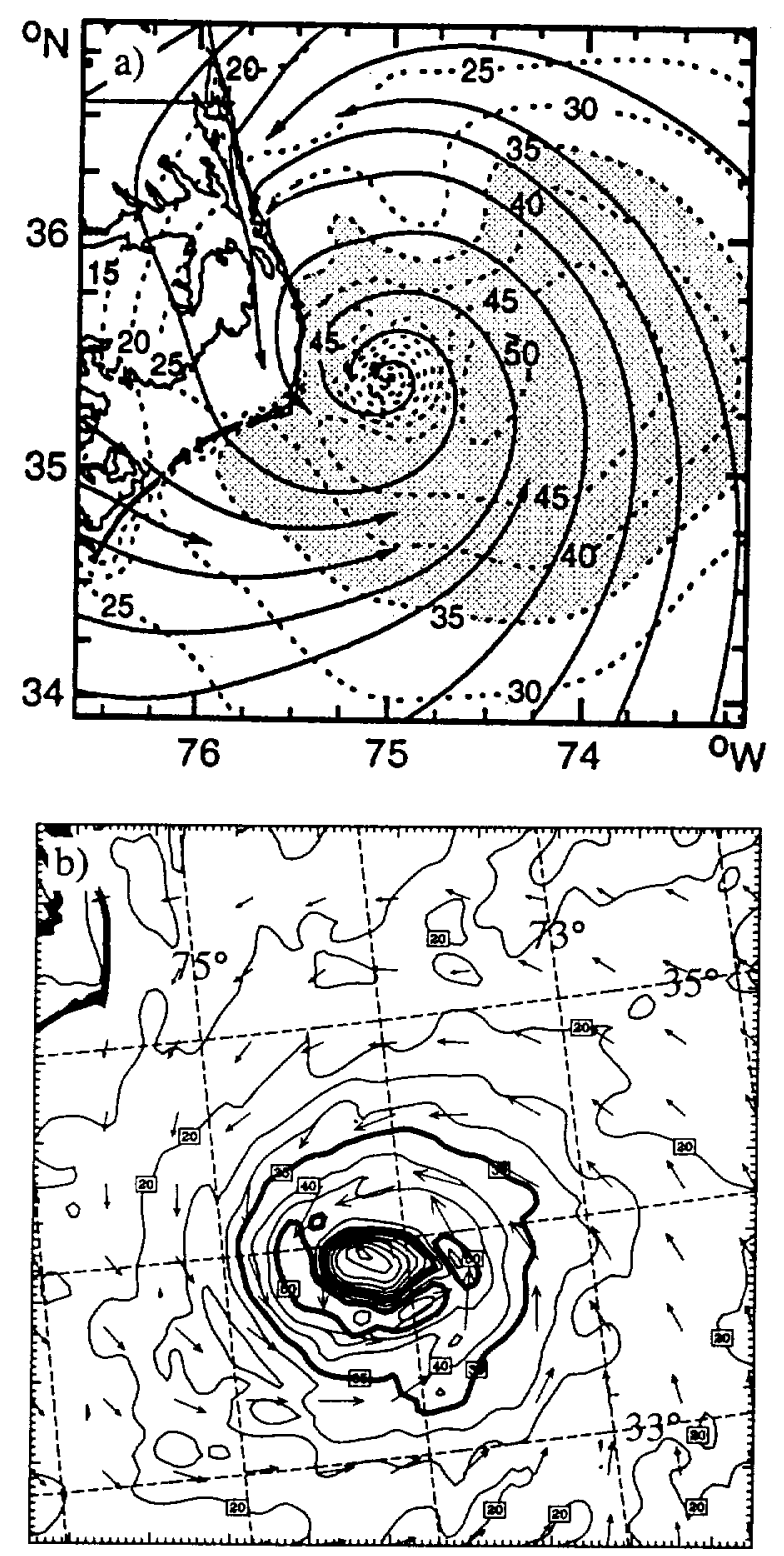

Figure 4. (a) Surface wind analysis for Hurricane Bob at 0300 UTC 19 August (courtesy of S. Houston, NOAA/HRD). Contours are isotachs drawn every $5 \mathrm{~m}$ $\mathrm{s}^{-1}$. Shaded region encloses winds exceeding $35 \mathrm{~m} \mathrm{~s}^{-1}$. (b) Simulated winds at the lowest model level $(42 \mathrm{~m})$ at $t=72 \mathrm{~h}$ valid at 0000 UTC 19 August. Contours are isotachs at $5 \mathrm{~m} \mathrm{~s}^{-1}$ intervals. Thick solid lines correspond to winds speeds of 35 and $50 \mathrm{~m} \mathrm{~s}^{-1}$. 


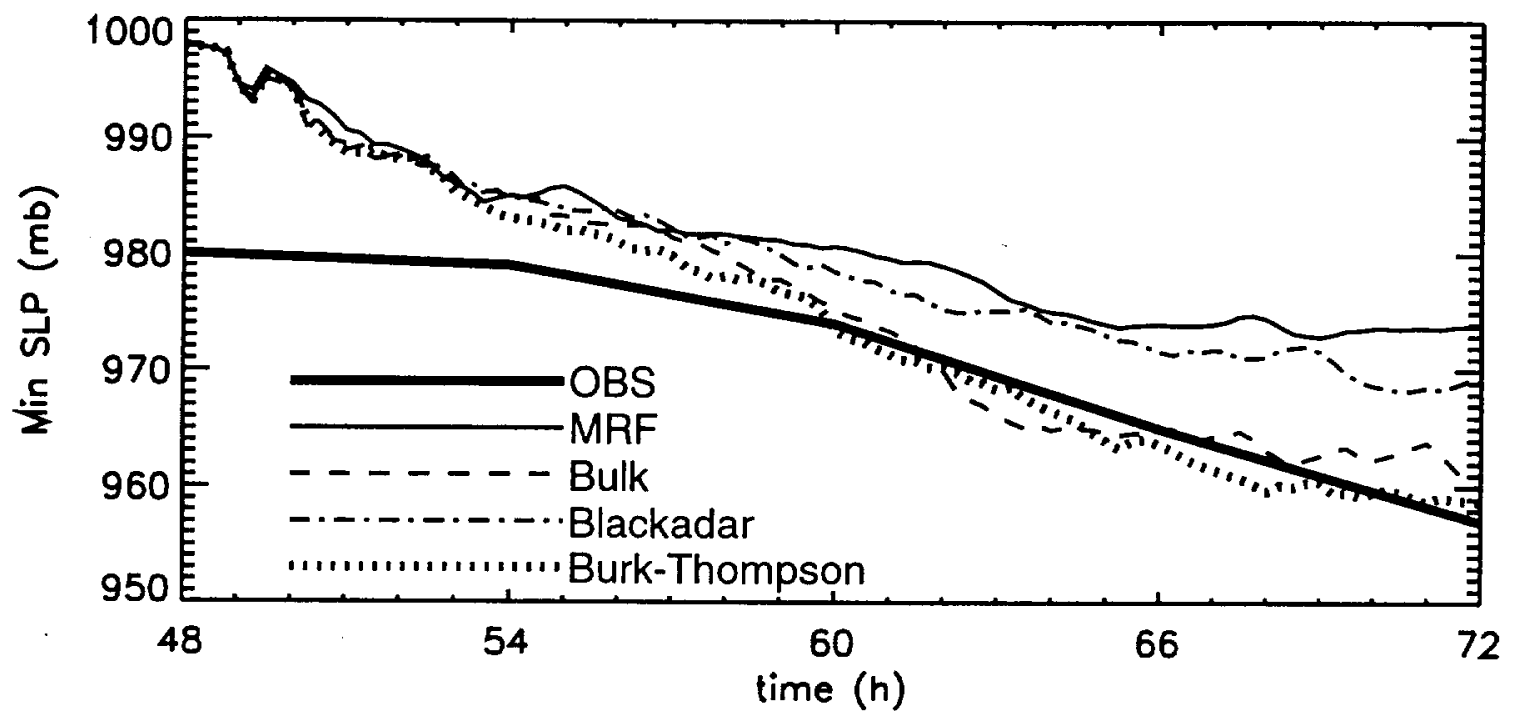

Figure 5. Time series of central pressure for the 24-h period of the high-resolution simulations. Comparison of the observed central pressure with values from the highresolution simulations with variable PBL physics. 
a) Burk-Thompson

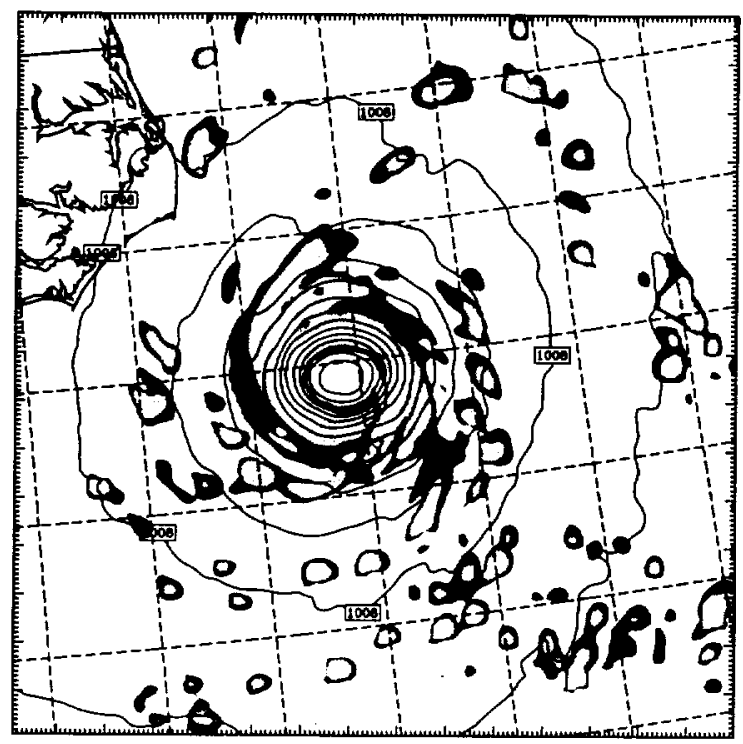

c) Blackadar

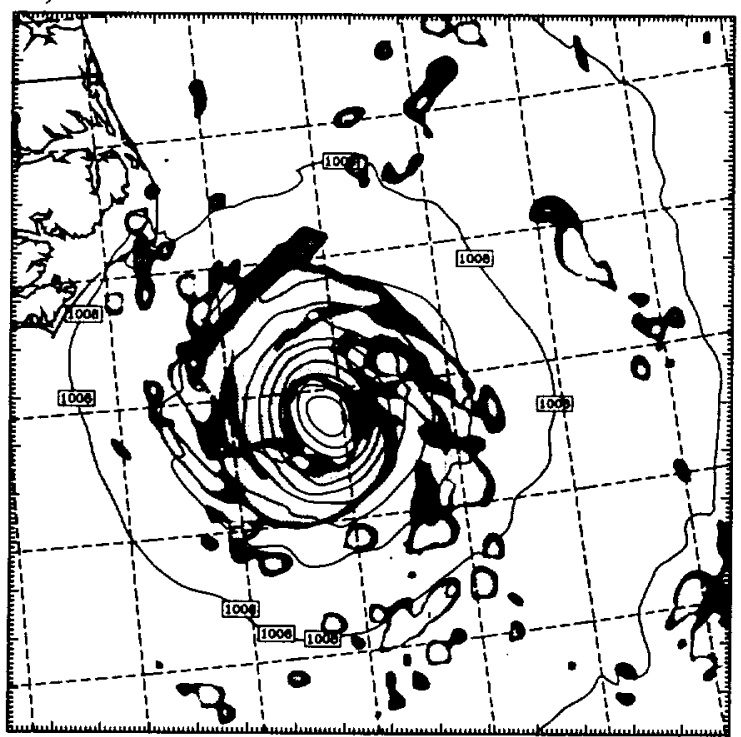

b) Bulk-aerodynamic

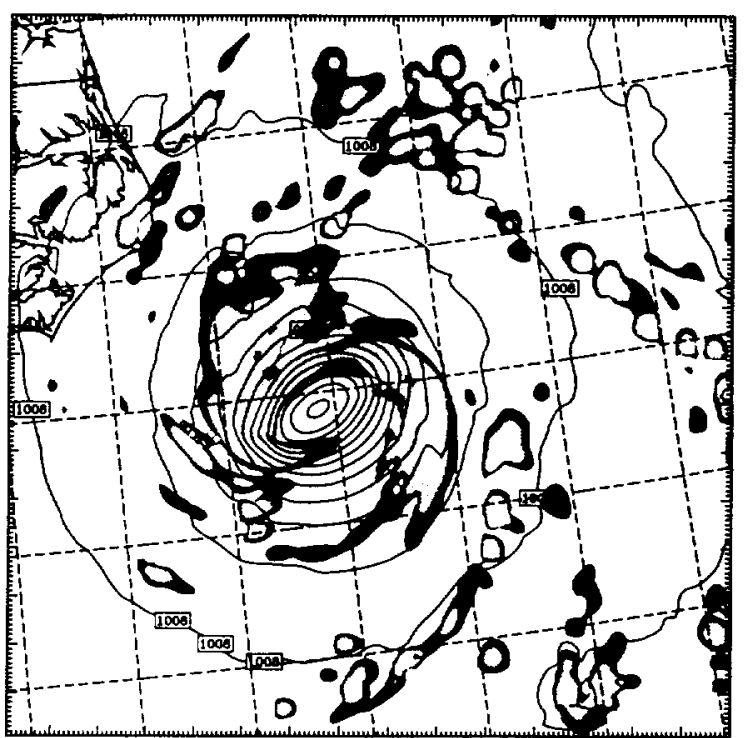

d) MRF

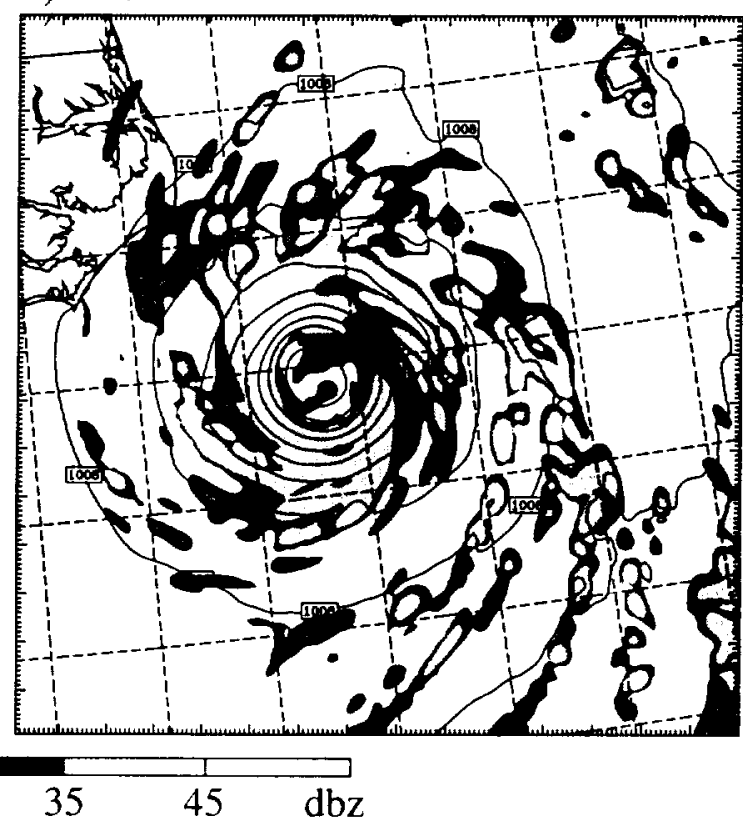

Figure 6. Simulated radar reflectivity patterns at $1 \mathrm{~km} \mathrm{MSL}$ and sea-level pressure at $t=72 \mathrm{~h}$ (valid at 0000 UTC 19 August) for the high-resolution simulations with variable PBL physics. Sea-level pressure contours are drawn every $4 \mathrm{mb}$. 

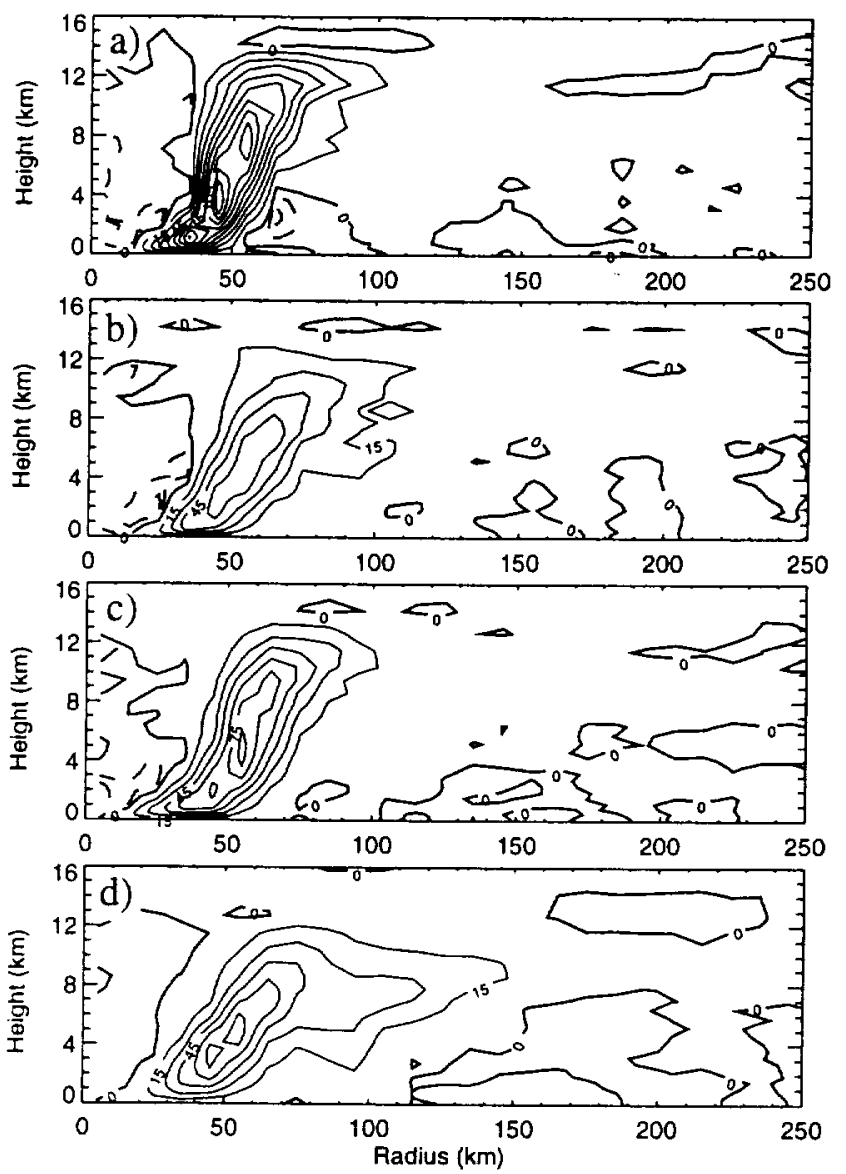

Figure 7. Vertical cross sections of azimuthally and temporally averaged vertical velocity averaged over hours 60-66, using output at 15 -min intervals. Positive values are contoured at intervals of $0.15 \mathrm{~m}$ $\mathrm{s}^{-1}$ (contour labels are in $\mathrm{cm} \mathrm{s}^{-1}$ ), negative values at intervals of $0.05 \mathrm{~m} \mathrm{~s}^{-1}$. Panels correspond to the following PBL schemes: (a) Burk-Thompson, (b) bulk-aerodynamic, (c) Blackadar, and (d) MRF. 

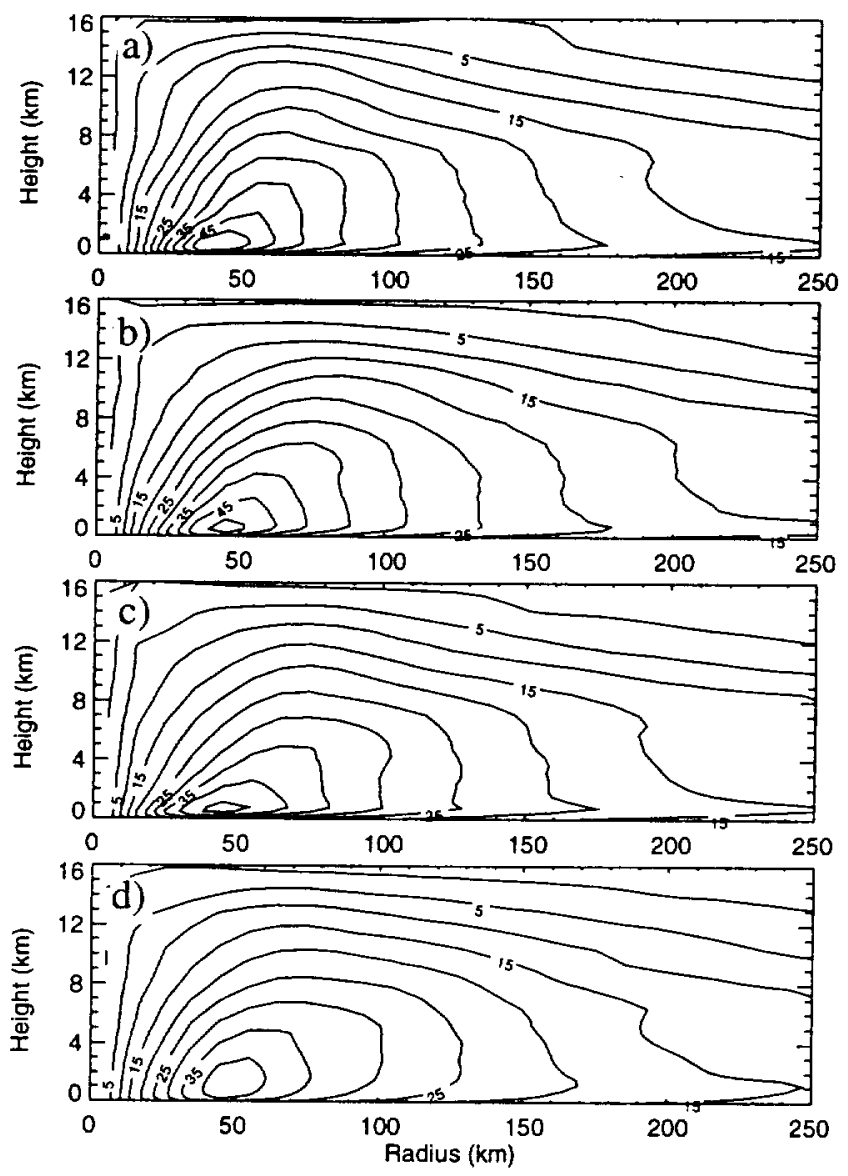

Figure 8. Same as in Fig. 7, but for tangential velocity. The contour interval is $5 \mathrm{~m} \mathrm{~s}^{-1}$. 

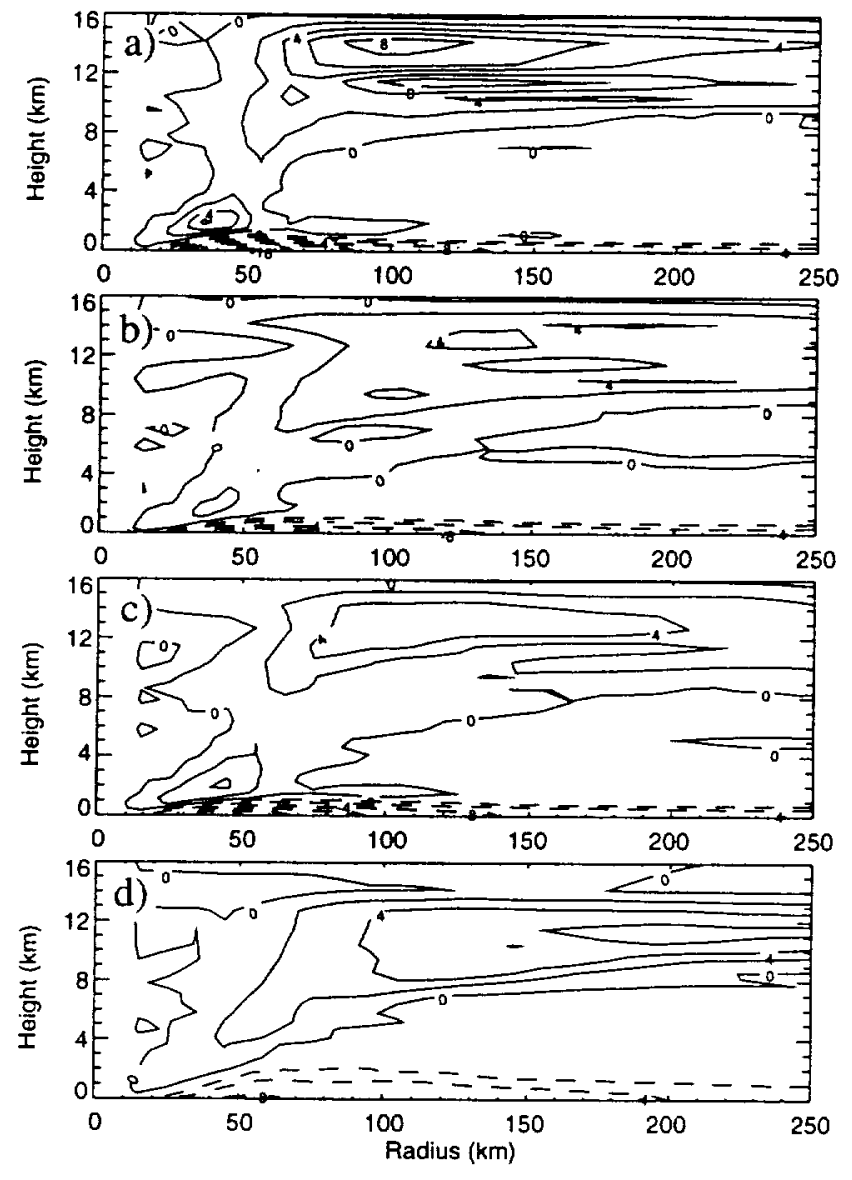

Figure 9. Same as Fig. 7, but for radial velocity. Positive values are contoured at intervals of $2 \mathrm{~m} \mathrm{~s}^{-1}$, negative values at intervals of $4 \mathrm{~m} \mathrm{~s}^{-1}$. 

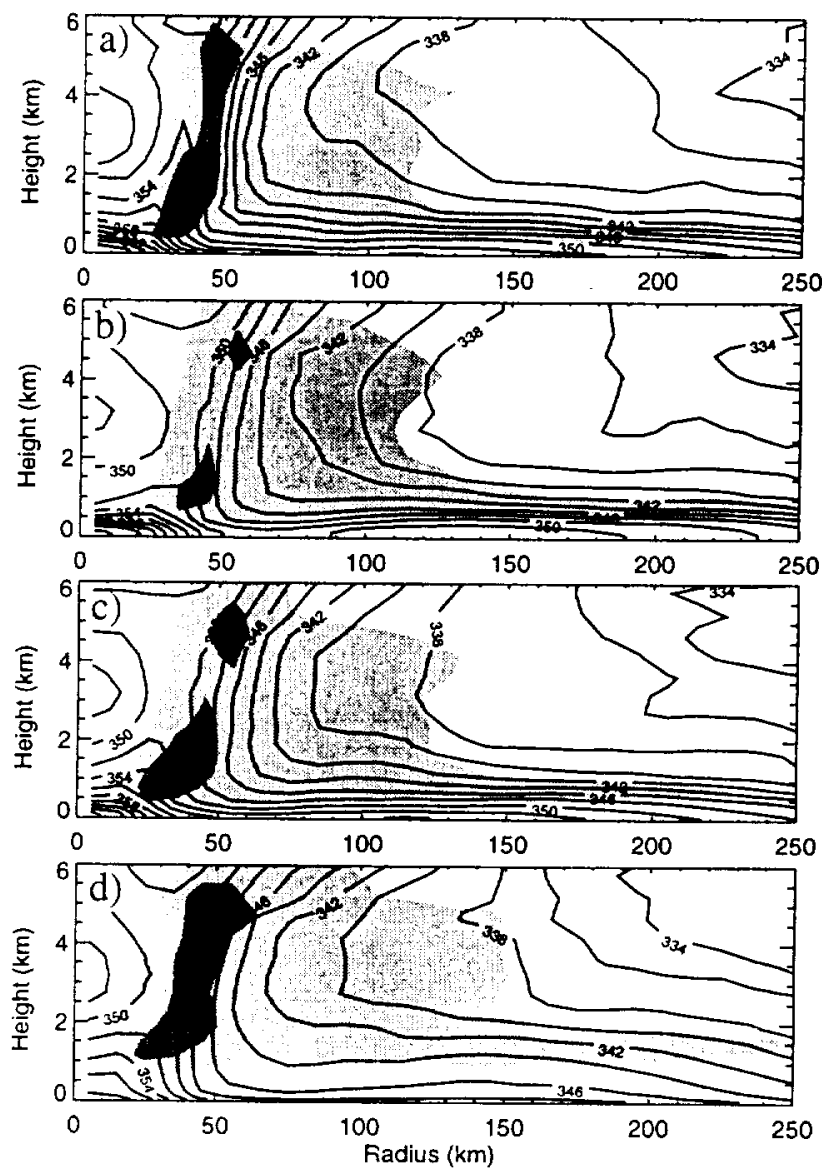

Figure 10. Same as Fig. 7, but for equivalent potential temperature and cloud water. Contours for $\theta_{e}$ are drawn at $2 \mathrm{~K}$ intervals. Cloud water amounts are indicated by the stippling, with light and dark stippling indicating values greater than 0.05 and $0.25 \mathrm{~g} \mathrm{~kg}^{-1}$, respectively. 

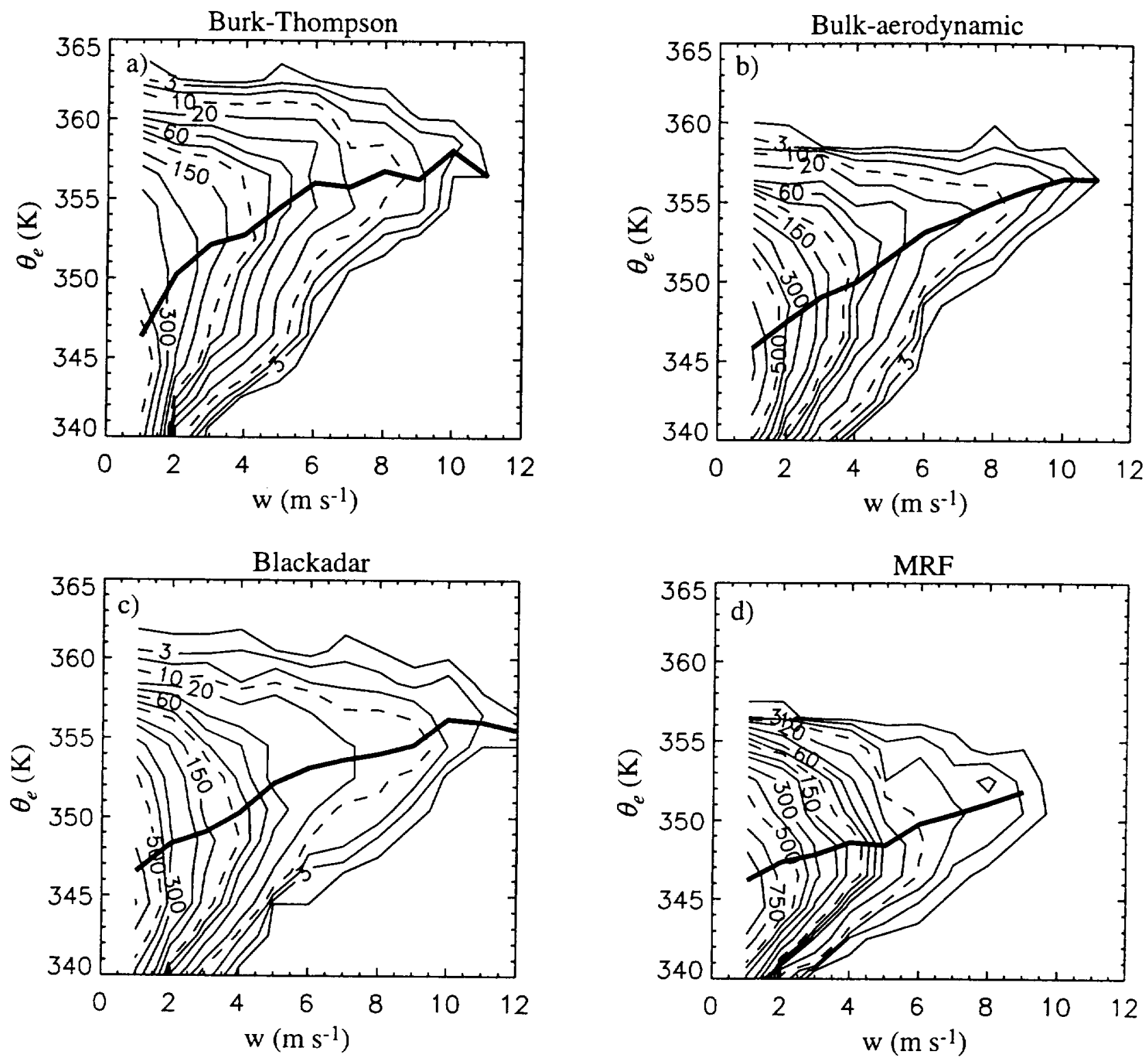

Figure 11. Contoured frequency diagrams showing the frequency of a given $\theta_{e}$ value occurring with a given vertical velocity, $w$. Contours show the number of grid points with the given $w$ and $\theta_{e}$ and are drawn at values of $1,3,6,20,40,60,80,150,200,300,400,750,1000$. The thick solid line indicates the average $\theta_{e}$ for a given $w$. Panels corresponds to the following PBL schemes: (a) BurkThompson, (b) bulk-aerodynamic, (c) Blackadar, and (d) MRF. 

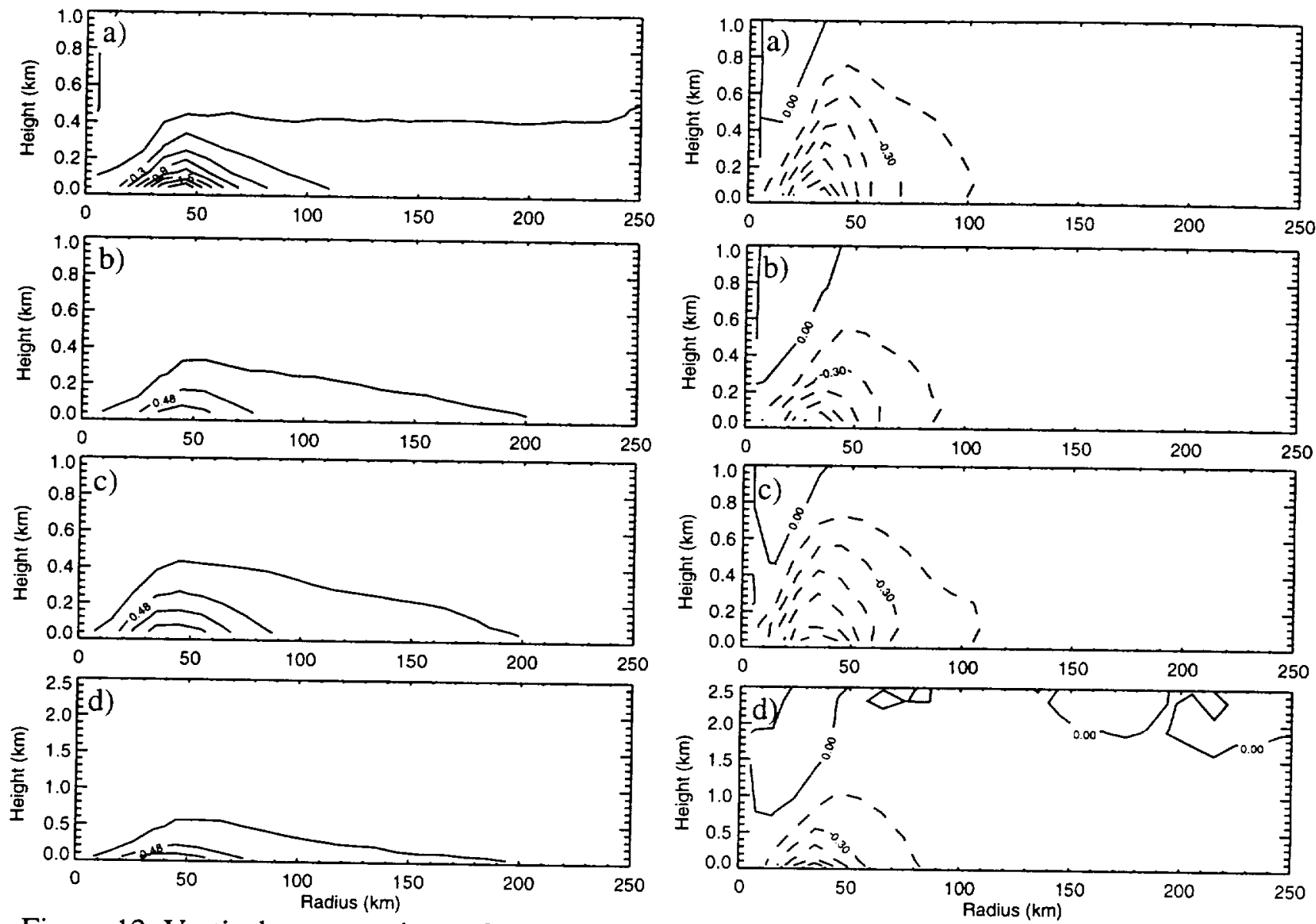

Figure 12. Vertical cross sections of azimuthally and temporally averaged radial velocity tendency, averaged over hours 62-66, using output at 2-h intervals. Contours are drawn at $0.4 \mathrm{~m} \mathrm{~s}^{-2}$. Panels Figure 13. Same as in Fig. 12, but for tangential velocity tendency. Contours are drawn at $0.15 \mathrm{~m} \mathrm{~s}^{-2}$.

correspond to the following PBL schemes: (a)

Burk-Thompson, (b) bulk-aerodynamic, (c)

Blackadar, and (d) MRF. Note that the vertical scale in (d) is $2.5 \mathrm{~km}$ compared to $1 \mathrm{~km}$ in the other panels. 

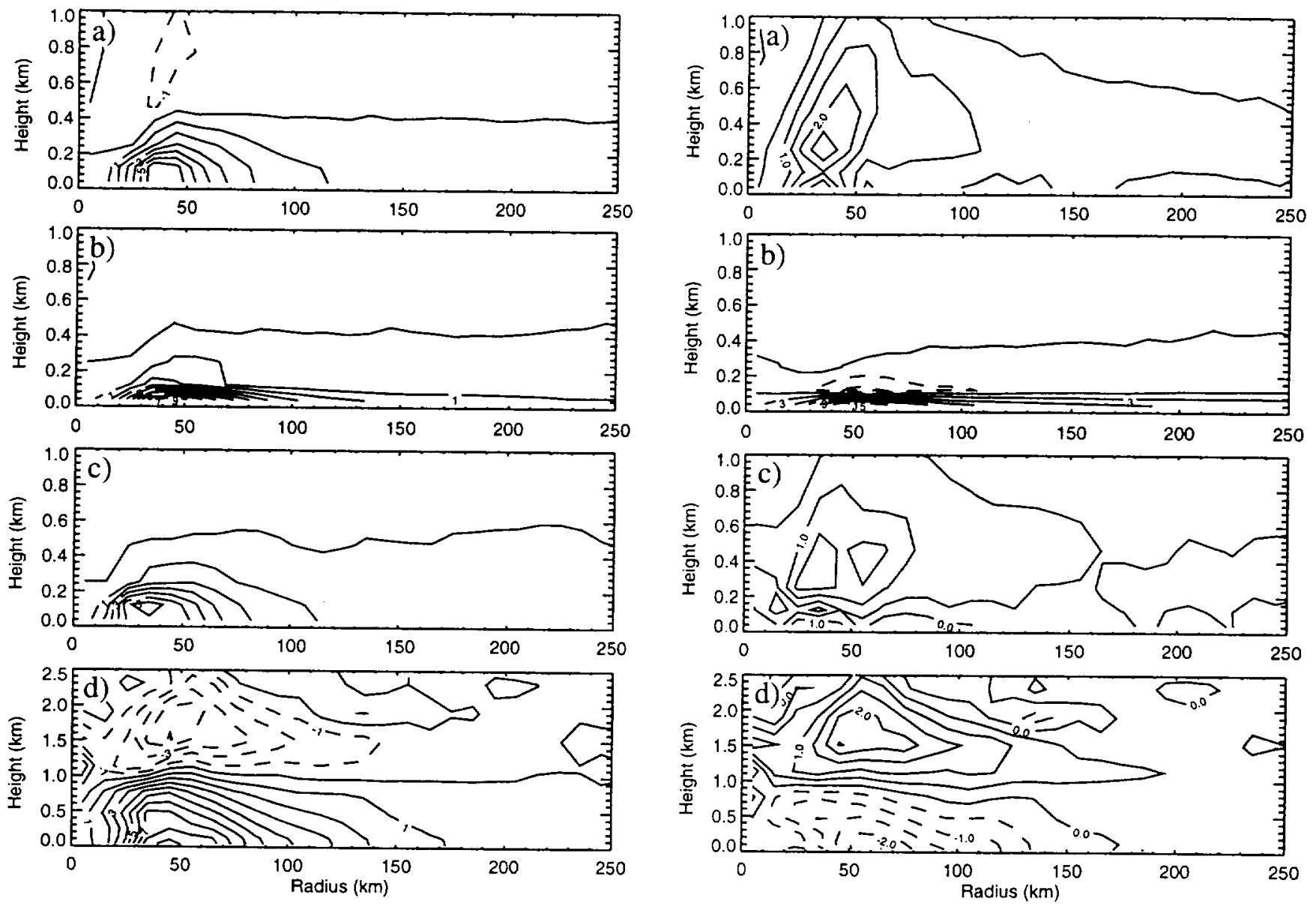

Figure 14. Same as in Fig. 12, but for temperature tendency. Contours are drawn at $1 \mathrm{~K} \mathrm{~h}^{-1}$.

Figure 15. Same as in Fig. 12, but for water vapor tendency. Contours are drawn at $0.5 \mathrm{~g}$ $\mathrm{kg}^{-1} \mathrm{~h}^{-1}$ in (a, c, and d) and at $3 \mathrm{~g} \mathrm{~kg}^{-1} \mathrm{~h}^{-1}$ in (b). 


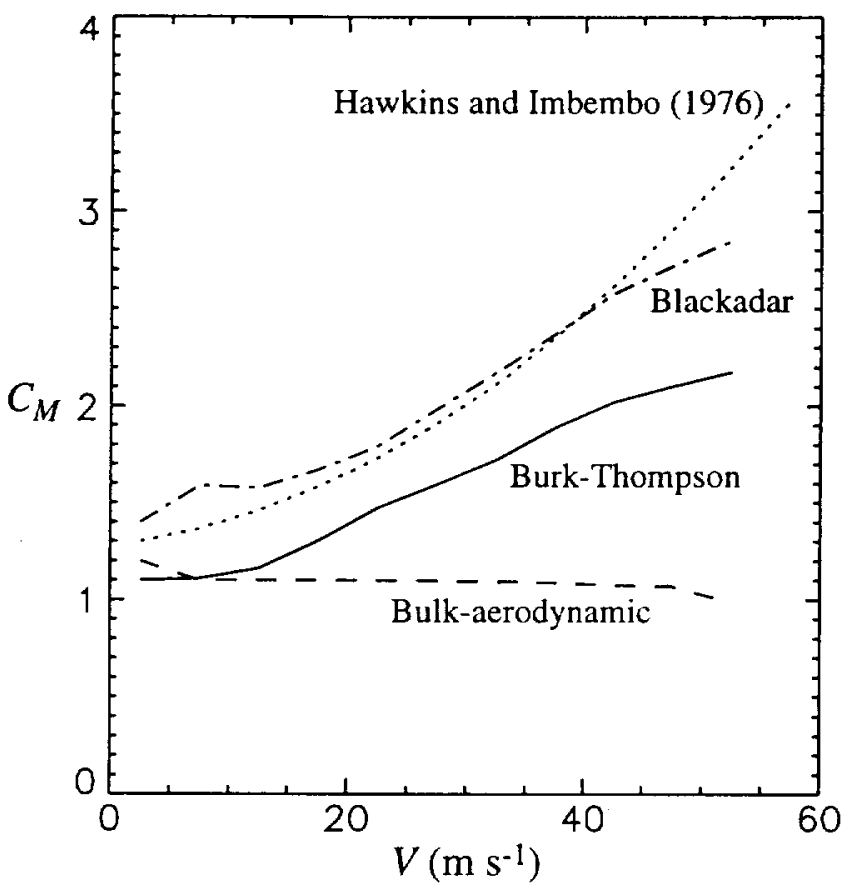

Figure 16. Exchange coefficients for momentum as a function of horizontal wind speed. 